\title{
A dynamical model of tumour immunotherapy
}

\author{
Federico Frascoli* \\ Department of Mathematics \\ Swinburne University of Technology \\ VIC, Australia \\ Peter S. Kim \\ School of Mathematics and Statistics \\ University of Sydney \\ NSW, Australia \\ Barry D. Hughes \\ Department of Mathematics and Statistics \\ The University of Melbourne \\ VIC, Australia \\ Kerry A. Landman \\ Department of Mathematics and Statistics \\ The University of Melbourne \\ VIC, Australia
}

\begin{abstract}
A coupled ordinary differential equation model of tumour-immune dynamics is presented and analysed. The model accounts for biological and clinical factors which regulate the interaction rates of cytotoxic $\mathrm{T}$ lymphocytes on the surface of the tumour mass. A phase plane analysis demonstrates that competition between tumour cells and lymphocytes can result in tumour eradication, perpetual oscillations, or unbounded solutions. To investigate the dependence of the dynamic behaviour on model parameters, the equations are solved analytically and conditions for unbounded versus bounded solutions are discussed. An analytic characterisation of the basin of attraction for oscillatory orbits is given. It is also
\end{abstract}

\footnotetext{
${ }^{*}$ Corresponding author

Email address: ffrascoli@swin.edu.au (Federico Frascoli)
} 
shown that the tumour shape, characterised by a surface area to volume scaling factor, influences the size of the basin, with significant consequences for therapy design. The findings reveal that the tumour volume must surpass a threshold size that depends on lymphocyte parameters for the cancer to be completely eliminated. A semi-analytic procedure to calculate oscillation periods and determine their sensitivity to model parameters is also presented. Numerical results show that the period of oscillations exhibits notable nonlinear dependence on biologically relevant conditions.

Keywords: Cancer, Immunotherapy, Ordinary differential equations, Dynamical systems, Finite-time tumour elimination

\section{Introduction}

Recent advances have opened the possibility of treating some cancers by developing preventative vaccines [1-4]. Such cancer vaccines would function by training a person's cytotoxic T lymphocyte (CTL) response to recognise and eliminate early-stage tumours close to inception, by producing a memory CTL population against certain tumour-associated antigens [5].

A challenge to designing preventative cancer vaccines is understanding the tumour-CTL dynamics that lead to successful tumour elimination by the CTL response. A recent mathematical modelling paper of Kim and Lee [6] engages this problem by formulating a hybrid partial differential equation (PDE) and agentbased model (ABM) of the dynamics of an anti-cancer CTL response in the vicinity of a developing tumour and the draining lymph node. That work points out the importance of tumour geometry in determining CTL effectiveness and the likelihood of eliminating the tumour. In particular, it observes that lymphocytes might eliminate the tumour mass at a rate proportional to the surface area rather than the volume of the tumour.

Here we formulate a simplified deterministic model of the tumour-CTL dynamics as a system of ordinary differential equations (ODEs). Our ODEs incorporate interactions between CTLs and tumour cells at a rate proportional to the surface area of a spherical tumour and are able to capture the dynamics of tumour-CTL oscillations. Although clinical evidence for oscillatory dynamics between solid tumours and immune cells is not yet thoroughly established, there are indications of such behaviours for systemic diseases such as leukaemia [7-9], and oscillations in both cancer and immune cells numbers have been observed in some clinical contexts [10-12]. Elimination of the cancer mass in finite time and 
oscillations are instead some of the typical findings in mathematical modelling of immunotherapies [13, 14], and, in particular, have been observed in the framework developed by Kim and Lee [6]. One of the advantages of the equations we propose over ABM formulations is that the ODEs in the current work are analytically tractable. This enables us to characterise dynamics mathematically over a wide range of parameters and obtain explicit mathematical descriptions of clinically relevant scenarios for tumour-CTLs interactions. In addition, the equations can be readily extended to consider alternative tumour geometries, partial penetration of $\mathrm{T}$ cells into the tumour mass, and other tumour growth rates.

Interest in mathematical modelling of tumour-immune dynamics has grown rapidly in recent years and various modelling approaches have been used to describe these phenomena. In particular, a large body of tumour-immune models has been developed using ODEs [15-22] and PDEs [23]. For the interested reader, a review of ODE models of tumour-immune interactions can be found in Eftimie et al. [14], whereas Bellomo et al. [24] and Roose et al. [25] contain thorough discussions of ODE and PDE models of tumour growth. A recent approach has focused on ABMs, or cellular automata, sometimes coupled with differential equations, to simulate tumour growth [26, 27], tumour growth and angiogenesis [28, 29], and tumour growth in the presence of an immune response [30-32]. While these models consider chemotherapy, immunotherapy, and other treatments against existing tumours, our equations focus on protective anti-tumour immunity by CTLs that would detect a tumour very early in development and could induce complete tumour elimination.

The paper is organized as follows. In Section 2, the coupled ODE system describing the dynamics of tumour and CTL populations is presented. A phase plane analysis is conducted in Section 3 and conditions that lead to bounded or unbounded solutions (as well as a degenerate case) are ascertained. In Section 4, analysis of the system leads to explicit solutions in terms of the Lambert $W$ function, leading to the determination of basins of attraction for perpetually oscillating solutions and for tumour elimination. Furthermore a semi-analytic procedure to determine the period of oscillations in terms of parameters and initial conditions is discussed. Numerical computations of periods as functions of a variety of clinically relevant factors are performed, to illustrate model sensitivity. Finally, in Section 5 conclusions are drawn and directions for future work are discussed. 


\section{Model equations}

We consider a tumour of volume $V(t)$. Although the volume changes with time, we assume that the shape is constant, so that the surface area of the tumour is $\rho V(t)^{2 / 3}$, where the dimensionless shape factor $\rho$ is given by $\rho=(36 \pi)^{1 / 3}$ for the case of a sphere, which is used in all our numerical computations. We assume that the number $N(t)$ of CTLs available to attack the tumour has a growth rate proportional to the current surface area of the tumour and a constant death rate, so that we have

$$
\frac{d N}{d t}=\rho r_{\mathrm{c}} V(t)^{2 / 3} N(t)-d_{\mathrm{c}} N(t) .
$$

The constant parameters $r_{\mathrm{c}}$ and $d_{\mathrm{c}}$ are the recruitment and death rates of CTLs, respectively. (Note, we refer to $r_{\mathrm{c}}$ as a rate although it is a rate per unit area.)

Concerning the interpretation of the first term on the right, we assume here that CTLs interacting with the tumour surface release chemokines to recruit other similar cells to the cancer site $[33,34]$.

We assume that the tumour has no vascular system so its growth is regulated by nutrient supply at the surface, with a volumetric growth rate proportional to the surface area. In the presence of CTLs, the tumour loses mass at a rate proportional to the area of its surface that is under attack from leukocytes. We introduce $C(t)$, a dimensionless function that we shall call the coverage, which is the fraction of the surface area that is under attack. Then we have

$$
\frac{d V}{d t}=\rho r_{\mathrm{t}} V(t)^{2 / 3}-\rho k V(t)^{2 / 3} C(t) .
$$

The constant parameters $r_{\mathrm{t}}$ and $k$ represent the tumour growth rate and the killing rate due to CTLs. (Similarly, $r_{\mathrm{t}}$ and $k$ are referred to as rates although they involve a length scale as well.) As it will be shown explicitly in Appendix A, the choice of a term proportional to $V(t)^{2 / 3}$ corresponds to a linear growth rate for the radius of the tumour. This is one of the proposed laws for the evolution and development of cancers [35], although the existence of a universal rule and its functional form is controversial and difficult to evaluate [36]. Although we have not considered vascular tumours, for which the T-cells are certainly able to access the tumour interior, we note that some penetration of an avascular tumour by T-cells is plausible. In Appendix B we discuss, as an interesting limiting case, an adjusted model in which the tumour loses mass at a rate proportional to its volume, corresponding to deep penetration of the tumour by the attacking T-cells.

We now assume that the fractional coverage of the surface area of the tumour is directly proportional to the concentration of T-cells delivered in the vicinity 
of the cancer. This concentration is also taken to be proportional to the total Tcell supply. If the local concentration of lymphocytes is sufficiently high, the tumour surface is under complete attack (i.e. completely covered) and no further T-cells can be recruited to the surface. Absorbing all proportionality constants, we introduce $\mathscr{N}$, which is the minimum total cell number in the vicinity of the cancer so that the tumour is under complete attack.

It follows that when $N(t)$ is too low for total coverage of the tumour to be achieved $(N(t)<\mathscr{N}$, say), we have $C(t)=N(t) / \mathscr{N}$. As originally defined, $C(t) \leq 1$. However, if we extend the definition to $C(t)=N(t) / \mathscr{N}$ for all values of $N(t)$, then $C(t)$ satisfies the same linear ODE as $N(t)$ and we arrive at the following model for the interaction of the CTLs and the tumour:

$$
\begin{aligned}
& \frac{d V}{d t}=\rho r_{\mathrm{t}} V(t)^{2 / 3}-\rho k V(t)^{2 / 3} \min \{1, C(t)\}, \\
& \frac{d C}{d t}=\rho r_{\mathrm{c}} V(t)^{2 / 3} C(t)-d_{\mathrm{c}} C(t) .
\end{aligned}
$$

Note that, in the following, given its definition as an extension of the coverage for $N(t) \geq \mathscr{N}$, we will also refer to $C(t)$ as the (dimensionless) CTL concentration.

Biologically relevant ranges and units of measurements for parameters and variables used in this model are given in Table 1 . The range of tumour volumes that our model can describe is assumed to be between 0 and $4 \times 10^{6} \mu \mathrm{m}^{3}$, corresponding to a tumour mass of approximately 0 to 1000 tumour cells, if we consider that tumour cells have a diameter of about $10 \mu \mathrm{m}[20,28,31,37]$.

Although our study could be generalized to consider different types of tumours, much experimental work has focused on breast cancer, e.g. Refs. [38-41], so we estimate our tumour growth rates from these data sets. Specifically, Spratt et al. calculate that the initial tumour cell doubling time is between 30 and 4800 days ( $\sim 13$ years) [40]. Weedon-Fekjær et al. find similar doubling times of 1.2 months to 6.3 years [41]. Other experimental studies report moderate doubling times of around 100 days $[38,39]$. Consequently, we use a maximum growth rate of $r_{\mathrm{t}}=0.5 \mu \mathrm{m} \cdot$ day $^{-1}$, which means that the tumour mass will grow by one cell diameter $(\sim 10 \mu \mathrm{m}$ as stated above) over a period of 10/0.5 $=20$ days, or approximately one month. We allow the growth rate to vary from zero to this maximum rate.

For the purposes of investigating tumour and CTL dynamics, we assume that the tumour killing rate of CTLs, $k$, is comparable to tumour growth rates. On the other hand, if CTL killing rates far outpaced tumour growth rates or vice versa, the system would result in very robust dynamics of rapid tumour death or 
uncontrollable growth, respectively. As a result, we assume that $k$ varies over the same range as $r_{\mathrm{t}}$, namely 0 to $0.5 \mu \mathrm{m} \cdot$ day $^{-1}$.

CTL recruitment rates are hard to estimate directly, but experimental studies have shown that this process can happen very effectively $[2,3]$, so we estimate a maximum rate of $r_{\mathrm{c}}=0.2 \mu \mathrm{m}^{-2} \cdot$ day $^{-1}$. If we assume that one CTL has a crosssectional area of approximately $100 \mu \mathrm{m}^{2}$, (because we assume a diameter of $\sim 10$ $\mu \mathrm{m}$ as above), then one CTL engaging the tumour can recruit additional cells at maximum rate $0.2 \times 100=20 \cdot$ day $^{-1}$, which corresponds to approximately one additional cell per hour. This rate may be quite fast, since transit times of CTLs from draining lymph nodes to tissues is more likely on the order of several hours [37], so we vary this recruitment rate from zero to the maximum value of $0.2 \mu \mathrm{m}^{-2}$ day $^{-1}$ to consider a range of possible values.

The half-life of CTLs has been experimentally estimated to be about 41 hours, which corresponds to a death rate of $d_{c}=0.4 \cdot$ day $^{-1}$ [42]. For our analysis, we allow this parameter to vary from 0 to 0.5 day $^{-1}$.

It is important to remark that not all solutions associated to Eqs. (1) and (2) have biological meaning, e.g. the cases of a negative tumour volume or a negative cell concentration are unphysical. A similar occurrence is also present in other well-established two-dimensional ODEs approaches to immunotherapies, for example in the model by Sotolongo-Costa et al. [43]. Thus, if the volume of the tumour becomes zero at some time $t^{*}$, that is $V\left(t^{*}\right)=0$ with $\frac{d V}{d t}\left(t^{*}\right) \leq 0$, then for all subsequent times $t>t^{*}, V(t)=0$. For this case, Eq. (2) reduces to $C(t)=C\left(t^{*}\right) e^{-d_{\mathrm{c}}\left(t-t^{*}\right)}$ for $t>t^{*}$. Note that this solution corresponds to complete elimination of the tumour by the lymphocytes in finite time, and represents one of the three possible types of solutions for the model.

The other two possible solutions are given by the existence of perpetual oscillations of the two cell populations and by unbounded solutions. Examples of bounded solutions are presented in Fig. 1. Unbounded $V(t)$ and $C(t)$ represent cases where the lymphocyte response fails to prevent the tumour from growing unrestrictedly, ultimately carrying the solution beyond biologically relevant ranges, and signifying a catastrophic clinical outcome. All three types of solutions based on model parameters are discussed in the following section, and their biological relevance, in the light of model assumptions, is examined in Sec. 5.

\section{Phase plane analysis of model equations}

The quantity that determines the outcome of CTL-tumour interactions is the ratio $r_{\mathrm{t}} / k$, that is, the tumour growth rate relative to the CTL killing rate. (The 
larger this ratio, the more aggressive the tumour). We see from Eq. (1) that irrespective of the value of $C(t)$,

$$
\frac{d V}{d t} \geq \rho\left(r_{\mathrm{t}}-k\right) V(t)^{2 / 3}
$$

Thus, if $r_{\mathrm{t}} / k>1$, the tumour growth is unbounded: even concomitant unbounded increase of the available CTL supply ensuring constant maximal attack of the tumour surface is unable to control the tumour. This is one of three scenarios that the phase plane analysis that follows reveals.

To understand the dynamics in the $(V, C)$-plane, let us first consider the nullclines. The solutions corresponding to $d C / d t=0$ in Eq. (2) are

$$
C=0 \quad \text { and } \quad V=\left(\frac{d_{\mathrm{c}}}{\rho r_{\mathrm{c}}}\right)^{3 / 2},
$$

irrespective of the value of $r_{\mathrm{t}} / k$. Nullclines corresponding to $d V / d t=0$ for Eq. (1) are

$$
V=0 \quad \text { and } \quad r_{\mathrm{t}}=k \min \{1, C(t)\} .
$$

If $r_{\mathrm{t}} / k>1$ the second of Eqs (4) has no solutions, but if $r_{\mathrm{t}} / k<1$ we have the nullcline

$$
C=\frac{r_{\mathrm{t}}}{k} \text {. }
$$

Examples of solutions for the bounded case at $r_{\mathrm{t}} / k<1$ are shown in Fig. 2 . Two of the nullclines intersect at a centre with coordinates

$$
\left(V_{\odot}, C_{\odot}\right)=\left(\left(\frac{d_{\mathrm{c}}}{\rho r_{\mathrm{c}}}\right)^{3 / 2}, \frac{r_{\mathrm{t}}}{k}\right) .
$$

Depending on the initial conditions $\left(V_{0}, C_{0}\right)$ at time $t=0$, two possible solutions exist: the tumour is destroyed and the concentration of lymphocytes goes to zero or the two populations oscillate perpetually, with respective maxima and minima that occur at different times. With no loss of generality, it is more mathematically convenient to consider initial conditions $\left(V_{0}, C_{0}\right)$ that belong to the region $0<C(t)<1$. In fact, in the next section we study analytically what conditions give rise to different types of solutions in this model, and what factors influence the success of the immune response. As stated, no unbounded solutions exist when a centre is present. The tumour can grow to a very large volume, but the immune response eventually catches up and prevents any trajectory from growing 
to infinity. This is a consequence of the assumption in our model that an endless supply of lymphocytes is available in the lymph node. In fact, there is no constraint on the maximal amount of CTL concentration $C(t)$ that can be present in the proximity of the tumour. The typical time evolutions for this scenario have been presented in Fig. 1.

It is possible to perform standard linear analysis [44] and find a closed expression for the frequency $\omega_{0}$ for small oscillations around the centre. This frequency is given by

$$
\omega_{0}=\left(\frac{2}{3}\right)^{\frac{1}{2}} \rho^{\frac{3}{4}}\left(r_{\mathrm{c}} d_{\mathrm{c}}\right)^{\frac{1}{4}} r_{\mathrm{t}}^{\frac{1}{2}} .
$$

Interestingly, this expression shows that small oscillations do not depend on the CTL killing rate $k$ and the contribution of other model parameters is regulated by different powers. Thus, for initial tumour sizes and T-cell concentrations in proximity of the centre, the efficiency of lymphocytes in destroying cancer cells is irrelevant for the period of oscillatory orbits. This does not mean, however, that an increase in the killing rate $k$ will be ineffective in eradicating the tumour. In fact, if $k$ is changed, then the value of $C_{\odot}$ is also altered, and the centre shifts position causing the system to follow a different trajectory. Importantly, small oscillations also depend on the shape factor $\rho$. For example, if parameters remain unaltered, a larger shape factor (i.e. a larger surface to volume ratio) makes small oscillations more frequent, since the CTL killing action depends on the amount of accessible tumour surface. In the case of spherical tumours considered in this paper, the expression for $\omega_{0}$ reduces to

$$
\omega_{0}=2 r_{\mathrm{t}}^{\frac{1}{2}}\left(\pi r_{\mathrm{c}} d_{\mathrm{c}}\right)^{\frac{1}{4}}
$$

The case of unbounded solutions is illustrated in Fig. 3. In this scenario, the absence of the nullcline $C=r_{\mathrm{t}} / k$ prevents the formation of a centre, and the nullcline $V=V_{\odot}$ is the locus of minima in $C(t)$. Considering initial conditions $\left(V_{0}, C_{0}\right)$ such that $V_{0}<V_{\odot}$, the solutions will initially decrease to a minimum in $C(t)$ and then grow without bound with increasing velocity. Clearly, when $r_{\mathrm{t}} / k>1$ the tumour grows faster than the rate at which it is destroyed by CTLs and cannot be eradicated, as shown by the time evolution in Fig. 4(a). Note also that the CTL concentration rapidly increases to unlimited values, as a result of a faster and faster recruitment, but the CTLs cannot contain or halt the expansion of the tumour.

In the final case (c) for $r_{\mathrm{t}}=k$, the rate of growth and the killing frequency are equal, so, when $C(t)$ reaches the threshold value of $C_{\max }=1$, the tumour 
ceases to enlarge and reaches its maximal volume (see Fig. 4(b)). Again, although the number of CTLs in the vicinity of the tumour grows without bound, they are not sufficient to kill the tumour, since we assume that only CTLs in contact with the tumour surface can kill its cells. The difference between this case and the unbounded case (b) is that in (b) both variables $V(t)$ and $C(t)$ eventually grow to infinity, whereas in the degenerate case only the CTL concentration is unbounded. In fact, when $C(t) \geq 1$ Eqs. (1)-(2) for $r_{\mathrm{t}}=k$ reduce to

$$
\begin{aligned}
& \frac{d V}{d t}=0, \\
& \frac{d C}{d t}=\rho r_{\mathrm{c}} V(t)^{2 / 3} C(t)-d_{\mathrm{c}} C(t),
\end{aligned}
$$

indicating that the tumour volume remains constant. Hence, although the dynamics for $0<C(t)<1$ are similar to the case of bounded orbits, when a solution crosses the nullcline $C=1$, its behaviour is regulated by the equations above, as exemplified in Fig. 5. The phase space now presents a degenerate centre, which possesses some of the features shown for the oscillatory case. In fact, the region $0<C(t)<1$ has the same properties. Note that the solution for $C(t) \geq 1$ has a different stability depending on its position with respect to the nullcline $V=\left(d_{\mathrm{c}} /\left(\rho r_{\mathrm{c}}\right)\right)^{3 / 2}$. The Jacobian always has one zero eigenvalue and a second eigenvalue $\lambda_{2}=-d_{\mathrm{c}}+\rho r_{\mathrm{c}} V(t)^{2 / 3}$, which is positive, and hence repelling, on the right side of the nullcline. This causes the degenerate solution to effectively perform only a half-turn around the centre, and go to infinity following a vertical line.

\section{Analytic results}

It is useful to consider whether analytic solutions exist for all the three cases: bounded, unbounded and degenerate. Besides being important from a theoretical point of view, existence of closed formulae in terms of model parameters are also valuable from a practical perspective. Understanding what combinations of parameters give rise to oscillations can help design more effective vaccination therapies that will respond successfully to a variety of characteristics of possible tumours.

\subsection{Solutions to model equations}

Given the $\min \{1, C(t)\}$ term in Eq. (1), it is evident that the case $C(t) \geq 1$ is solvable by direct integration. If $\min \{1, C(t)\}=1$, no coupling between $V(t)$ and 
$C(t)$ is present in Eq. (1), and the following expression for $V=V(t)$ can be found:

$$
V(t)=\frac{1}{27}\left(3 V_{I}^{1 / 3}-\left(k-r_{\mathrm{t}}\right) \rho t\right)^{3},
$$

where the initial condition for the tumour volume at $t=0$ is indicated by $V(0)=$ $V_{I}$. When solving for this case, $V_{I}$ is the initial value of $V$ at the boundary between the two regions that are relevant in the model, hence $C(0)=1$. This notation is used to distinguish the point $\left(V_{I}, 1\right)$ from a generic initial condition $\left(V_{0}, C_{0}\right)$ for the whole solution in the phase plane.

Note that the solution is a cubic, as in Kim and Lee [6], and its boundedness directly depends on the sign of the term $\left(k-r_{\mathrm{t}}\right)$. If $k>r_{\mathrm{t}}$, it eventually reaches zero in finite time (tumour eradication). Otherwise, the solution is either unbounded for $k<r_{\mathrm{t}}$ or constant and equal to $V_{I}$ for the degenerate case $k=r_{\mathrm{t}}$. Substituting Eq. (11) into Eq. (2) and integrating, we obtain:

$$
C(t)=\exp \left(-d_{\mathrm{c}} t+\frac{r_{\mathrm{c}} V_{I}}{k-r_{\mathrm{t}}}-\frac{r_{\mathrm{c}}\left(3 V_{I}^{1 / 3}-\left(k-r_{\mathrm{t}}\right) \rho t\right)^{3}}{27\left(k-r_{\mathrm{t}}\right)}\right) .
$$

As stated, given that $C(0)=1$, the above expression contains only the initial condition for $V(t)$, i.e. $V_{I}$. The exponent in the solution has a linear term with respect to time that is proportional to the decay rate of CTLs $d_{\mathrm{c}}$ and a cubic dependence from the evolution of the tumour volume. The remaining term of the exponent is inversely proportional to $\left(k-r_{\mathrm{t}}\right)$. In the degenerate case, $C(t)$ is given by $C(t)=\exp \left(-d_{\mathrm{c}} t+r_{\mathrm{c}} V_{I}^{2 / 3}\right)$. So, since $V(t)=V_{I}$ when $k=r_{\mathrm{t}}$, the degenerate solution grows to infinity following a vertical line in the phase plane (see Fig. 5). A graphic depiction of a family of solutions given by Eqs. (11)-(12) for the bounded and unbounded cases is presented in Fig. 6.

Solving for the case $0<C(t)<1$ is not straightforward, due to the presence of the bilinear term in Eq. (1). A strategy to arrive at analytic solutions requires substitutions that recast the model equations into a simpler system and is illustrated in the Appendix. Closed expressions for $V=V(t)$ and $C=C(t)$ can be found, although they are particularly involved. Instead, it is interesting to use the above mentioned strategy to obtain expressions for level set curves.

These are implicit functions such that $g\left(V(t), C(t), V_{0}, C_{0}\right)=0$, where $\left(V_{0}, C_{0}\right)$ are initial conditions at $t=0$ chosen in the region $0<C(t)<1$. As shown in the Appendix, a separable ordinary differential equation can be integrated to arrive at

$$
\frac{C(t)}{C_{0}} \exp \left(-\frac{k}{r_{\mathrm{t}}}\left(C(t)-C_{0}\right)\right)-\exp \left(\frac{r_{\mathrm{c}}}{r_{\mathrm{t}}}\left(V(t)-V_{0}\right)-\frac{3 d_{\mathrm{c}}}{\rho r_{\mathrm{t}}}\left(V(t)^{1 / 3}-V_{0}^{1 / 3}\right)\right)=0 .
$$


This expression can be inverted to obtain

$C(t)=-\frac{r_{\mathrm{t}}}{k} W_{(-1 ; 0)}\left(-\frac{C_{0} k}{r_{\mathrm{t}}} \exp \left\{-\frac{C_{0} k}{r_{\mathrm{t}}}\left[\frac{r_{\mathrm{c}}}{r_{\mathrm{t}}}\left(V(t)-V_{0}\right)-\frac{3 d_{\mathrm{c}}}{\rho r_{\mathrm{t}}}\left(V(t)^{1 / 3}-V_{0}^{1 / 3}\right)\right]\right\}\right)$.

In the solution (14), we write for brevity $W_{(-1 ; 0)}(x)$ to represent the two real branches of the Lambert $W$ function [45]. The Lambert $W$ function is defined implicitly by $W(x) \exp [W(x)]=x$. For $-1 / e<x<0$, this transcendental equation for $W(x)$ has two solutions, denoted by $W_{-1}(x)$ and $W_{0}(x)$, with $W_{-1}(x)<-1$ and $W_{0}(x)>-1$. The solutions coincide at $x=-1 / e$ and $W_{0}(x)$ is the solution that continues into the region $x \geq 0$. Examples of solutions obtained using Eq. (14) are presented in Fig. 7.

It is important to stress that, whilst for $r_{\mathrm{t}}<k$ both branches of the Lambert $W$ function contribute to the solution, in the unbounded and degenerate cases only the 0 branch is a solution. This means that in Fig. 7(a) the upper part of the egg-shaped curves corresponding to $C(t)>r_{\mathrm{t}} / k$ is described by the -1 branch, whereas the lower part for $C(t)<r_{\mathrm{t}} / k$ is given by the 0 branch. As the ratio $r_{\mathrm{t}} / k$ increases towards one, the $C$-coordinate of the centre $C_{\odot}$ increases, and the region of solutions belonging to the -1 branch gets smaller. At the degenerate stage, when $C_{\odot}=1$, the solutions for $0<C(t)<1$ are captured only by the 0 branch. This effect occurs because the expression for $C(t)$ corresponding to the branch $W_{-1}(x)$ in Eq. (14) is equal to one for $r_{\mathrm{t}}=k$, and is bigger than one for $r_{\mathrm{t}}>k$.

Clearly, the final, complete solution to the model equations will be given by the union of the solution for $0<C(t)<1$ with the solution for $C(t) \geq 1$, depending on the initial condition $\left(V_{0}, C_{0}\right)$. Examples of full solutions for different values of the ratio $r_{\mathrm{t}} / k$ are shown in Fig. 8.

\subsection{Basin of attraction for oscillatory solutions}

When the model equations give rise to a centre for $r_{\mathrm{t}} / k<1$, it is relevant to find out which initial conditions cause a solution to be of the oscillatory or eradication type. In Fig. 9, the basin of attraction for oscillatory solutions is illustrated. Rather than reasoning in terms of generic initial conditions $\left(V_{0}, C_{0}\right)$, it is algebraically simpler to consider the intersection of a generic solution with the line $C=1$. Indicating the $V$-coordinate at such intersection with $V_{I}$, it turns out that if $V_{I}$ is less than an extremal value $V_{I}^{*}$, the solution is oscillatory.

To prove this result, we consider the extremal solution for the case $C(t) \geq 1$, given by the cubic Eq. (11) with one intersection at the phase point $(V, C)=(0,1)$. This solution is labelled as extremal because it represents the quickest solution to 
tumour eradication. All other solutions having $C$-coordinates larger than one at $V=0$ are also eradication solutions, but $V$ reaches zero at longer times. Conversely, there are no solutions having $C$-coordinates smaller than one at $V=0$, because intersections with the line $C=1$ occurring at $V>0$ are typical of oscillatory solutions and obviously do not happen in the case of tumour eradication.

To find the extremal value $V_{I}^{*}$, we consider the time $t_{f}^{*}$ needed for the extremal orbit to reach the intersection $(V, C)=(0,1)$. According to Eq. (11),

$$
t_{f}^{*}=3 \frac{\sqrt[3]{V_{I}}}{\rho\left(k-r_{\mathrm{t}}\right)} .
$$

We substitute the above expression into the solution for $C(t)$ in Eq. (12) and find the relevant root for the cubic equation that results from imposing that $C\left(t_{f}^{*}\right)=1$. Of the three roots, one is zero, one is negative and is discarded, and one is positive. This last root provides the final result for $V_{I}^{*}$, i.e. the right edge of the boundary of the basin of attraction, which is given by

$$
V_{I}^{*}=3 \sqrt{3}\left(\frac{d_{\mathrm{c}}}{\rho r_{\mathrm{c}}}\right)^{3 / 2}=3 \sqrt{3} V_{\odot},
$$

where $V_{\odot}$ is the $V$-coordinate of the centre. To sum up, the extremal solution, i.e. the first eradication orbit, starts at coordinates $\left(V_{I}^{*}, 1\right)$ and ends at $(0,1)$. The total time taken for this trajectory is given by substituting $V_{I}^{*}$ in the previous expression for $t_{f}^{*}$. Any other solution whose $V$-coordinate at the intersection $V_{I}$ is on the right of $V_{I}^{*}$ is eradicated (see Fig. 9).

A solution can be oscillatory or not depending on its distance from the centre, when it intersects the boundary $C=1$. This is an interesting result, because it shows that there is a maximal size for tumours after which they are efficiently destroyed by the primed CTLs. Eq. (15) shows that a faster recruitment rate and a longer life for CTLs contribute to reduce that maximal size, making a vaccination response more efficient. Also, the dependence on the shape factor $\rho$ indicates that tumours with a smaller surface area to volume ratio have a larger basin of attraction for oscillatory orbits and the compactness of their shapes make cancers more resilient to attack by lymphocytes. The significance of this result in the light of the assumptions used for this model is discussed at length in Sec. 5.

\subsection{Period of oscillations}

Using the expression (14) for $C(t)=C(V(t))$, it is possible to illustrate a semianalytic procedure for investigating the periods of oscillatory solutions. The total 
period of any of such solutions is constituted by two or three terms, depending on whether the chosen orbit remains entirely within the region $0<C(t)<1$ or presents bounded excursions in the $C(t) \geq 1$ half-plane (see Fig. 10).

In the first case, the total period is the sum of the time spent when $C(t)<r_{\mathrm{t}} / k$ plus the analogous term for $C(t)>r_{\mathrm{t}} / k$. For $0<C(t)<1$, terms containing the branches $W_{0}(x)$ and $W_{-1}(x)$ are present. Using quadrature methods [46] and indicating with $V_{A_{1}}$ and $V_{B_{1}}$ the left and right intersections of the solution with the line $C=r_{\mathrm{t}} / k$ (see Fig. 10), the period for such a half turn below the centre is given by

$$
T_{0}=\rho r_{\mathrm{t}} \int_{V_{A_{1}}}^{V_{B_{1}}} \frac{V^{-\frac{2}{3}} d V}{1+W_{0}\left(\frac{C_{0} k}{r_{\mathrm{t}}} \exp \left\{-\frac{C_{0} k}{r_{\mathrm{t}}}\left[\frac{r_{\mathrm{c}}}{r_{\mathrm{t}}}\left(V-V_{0}\right)-\frac{3 d_{\mathrm{c}}}{\rho r_{\mathrm{t}}}\left(V^{1 / 3}-V_{0}^{1 / 3}\right)\right]\right\}\right)} .
$$

A similar expression for $T_{-1}$ with inverted ranges of integration gives the period for the other half turn above the centre. Note that $V_{A_{1}}$ and $V_{B_{1}}$ are solutions to a cubic equation given by imposing that $C(t)=r_{\mathrm{t}} / k$ and $V(t)=V_{I}$ in Eq. (13):

$$
-\frac{k}{r_{\mathrm{t}}}\left(\frac{r_{\mathrm{t}}}{k}-C_{0}\right)-\log \frac{r_{\mathrm{t}}}{k C_{0}}=\frac{r_{\mathrm{c}}}{r_{\mathrm{t}}}\left(V_{I}-V_{0}\right)-3 \frac{d_{\mathrm{c}}}{\rho r_{\mathrm{t}}}\left(V_{I}^{1 / 3}-V_{0}^{1 / 3}\right) .
$$

Closed expression for the roots are particularly involved, so we do not report them here, and depend on the initial conditions $V_{0}$ and $C_{0}$ in the region $0<C(t)<1$.

When the orbit is not fully contained within the region $0<C(t)<1$, the ranges of integration need to be substituted with the values of $V_{I}$ and $V_{F}$ at the intersection with the line $C=1$. These intersections can also be calculated using the expression for the level curves Eq. (13) and imposing that $C(t)=1$ and $V(t)=V_{I}$. Again, a cubic equation similar to the one previously discussed needs to be solved:

$$
-\frac{k}{r_{\mathrm{t}}}\left(1-C_{0}\right)-\log C_{0}=\frac{r_{\mathrm{c}}}{r_{\mathrm{t}}}\left(V_{I}-V_{0}\right)-3 \frac{d_{\mathrm{c}}}{\rho r_{\mathrm{t}}}\left(V_{I}^{1 / 3}-V_{0}^{1 / 3}\right) .
$$

As indicated in Fig. 10, we need to calculate integrals similar to Eq. (16) using the -1 branch, and evaluate the duration of the two trajectories $B_{2} \rightarrow I$ and $F \rightarrow A_{2}$. To complete the calculation, the time $T_{e}$ needed for a full, bounded excursion in the half-plane $C(t) \geq 1$ can be calculated using Eq. (12) and imposing the condition $C\left(T_{e}\right)=1$. Solving the cubic equation, the positive root is given by

$$
T_{e}=\frac{9 \rho r_{\mathrm{c}}^{1 / 2} V_{I}^{1 / 3}+3 \sqrt{3} \rho^{1 / 2}\left(4 d_{\mathrm{c}}-\rho r_{\mathrm{c}} V_{I}^{2 / 3}\right)^{1 / 2}}{2 \rho^{2} r_{\mathrm{c}}^{1 / 2}\left(k-r_{\mathrm{t}}\right)}
$$


where $V_{I}$, as noted, is the intersection with $C=1$ on the right side of the centre. A closed expression for the second intersection $V_{F}$ can also be found by considering Eq. (11) and substituting the above expression for $T_{e}$, i.e. using the expression for $V(t)$ in the region $C(t) \geq 1$ rather than the equation for the level set curves in the region $0<C(t)<1$.

Beyond semi-analytic expressions, numerical calculations can be employed to examine the variation of periods of oscillation with respect to model parameters. In Fig. 11, periods for orbits for different ratios $r_{\mathrm{t}} / k$ are plotted. Choosing the initial condition $C_{0}=9 / 10 C_{\odot}$, where $C_{\odot}$ is the C-coordinate of the centre for the chosen model parameters, it is possible to vary $V_{0}$ and compute the period of an oscillatory solution as a function of the distance from the centre $V_{\odot}$. As the ratio $r_{\mathrm{t}} / k$ approaches the degenerate value of 1 , the periods associated with initial conditions at the left, i.e. $V_{0} / V_{\odot}<1$, and on the right, i.e. $V_{0} / V_{\odot}>1$, of the centre increase in a nonlinear fashion. When the initial condition starts closer to the centre, i.e. $V_{0} / V_{\odot} \simeq 1$ the period approaches the limiting value $2 \pi / \omega_{0}$, corresponding to the regime of small oscillations. The shape of this trough depends on the value of $r_{\mathrm{t}} / k$, with an increasingly more marked V-shaped appearance as the ratio approaches one. Note that for each curve, the two arms meeting at the minimum are not symmetric and that the segments on the right of the extremum become almost linear as the distance from the centre grows larger. On the other hand, the segments on the left side rapidly rise towards high $T$, with concavities that depend on the ratio $r_{\mathrm{t}} / k$. All curves exist up to the limiting value $V_{0}$ such that the intersection of the solution $V_{I}$ with the line at $C=1$ is such that $V_{I}<3 \sqrt{3} V_{\odot}$, which corresponds to the edge of the basin of attraction discussed in Fig. 9.

Note that an increase in $d_{\mathrm{c}}$, corresponding to higher death rates for CTLs, alters the curves presented in Fig. 11 . The minimum for $2 \pi / \omega_{0}$ occurs at higher values and both arms around the minimum are shifted up, with a consequent increase in steepness. Clearly, if CTLs die at a faster rate with all other parameters staying constant, the tumour can grow to larger volumes and the cycles between the two competing populations of cells take longer to complete.

It is also interesting to understand how larger recruitment rates $r_{\mathrm{c}}$ affect the periods of oscillation. In Fig. 12, the shapes of curves and the minima for $V(0) / V_{\odot} \rightarrow$ 1 resemble the previous example for varying $k$. In this case though, the minima occur at different periods, since the frequency of small oscillations $\omega_{0}$ depends on $r_{\mathrm{c}}$. Quicker rates of recruitment decrease the period of oscillations, with an effect that depends nonlinearly on the initial tumour volume. Changes in $r_{\mathrm{c}}$ alter the periods more dramatically than changes in $k$. It should also be noted that, in general, the extension of the region for which the curves in Fig. 12 exist directly 
depends on the value of $r_{\mathrm{c}}$. In fact, because the basin of attraction for oscillatory solutions is bounded by $3 \sqrt{3} V_{\odot}$ and $V_{\odot} \sim r_{\mathrm{c}}^{-3 / 2}$, the length of the basin is inversely proportional to the rate of recruitment. This dependence agrees with the intuition that faster recruitment processes increase the effectiveness of the CTL response against the tumour, promoting eradication.

\section{Conclusions}

We develop and analyse a two-dimensional ODE model of CTL-tumour dynamics that accounts for tumour geometry in determining interaction rates between the two populations of cells. Our model provides an analytically tractable system. Furthermore, it reproduces the phenomena of oscillatory behaviour and finite-time tumour elimination obtained in more computationally complex approaches, such as that of Kim and Lee [6]. Indeed, our results show that the oscillations are inherent to the dynamics of the system and not due to the stochastic nature of the computational model used by Kim and Lee [6].

In agreement with the findings in that paper, the proposed equations do not admit a "small tumour size" equilibrium, whereas other models existing in the literature present such a solution [13, 14]. In fact, our system of ODEs does not contain any assumption about a possible inhibition of leukocytes' recruitment rates or a deterioration in CTLs killing efficiently due to an anti-immune response by cancer cells. Other two-dimensional approaches that focus on immunotherapies for tumours in later stages of development do include a term that weakens the response of the immune system as the tumours grows larger [17, 43, 47, 48]. The interplay between the pro-immune and anti-immune terms gives rise to a balance between the two populations, corresponding to a small tumour size equilibrium.

For Eqs. (1)-(2), a degenerate solution has been found in the case of a tumour growing at the same rate at which its cells are destroyed by CTLs. Although the tumour size remains constant, this solution is not stable since the population of T-cells grows to infinity in an unphsical way. This is a consequence both of the absence of the anti-immune response term, which could block the increase in cell concentration to large numbers, and of an unlimited and constant influx of leukocytes from the lymph node, which acts as an infinite reservoir for T-cells.

In fact, our model contains a number of hypotheses that are advantageous from the mathematical point of view, but that have to be taken into consideration when relating our results to clinical practice. First of all, the tumour mass is treated as continuous, so, in principle, tumours of very small size can regrow. This is evident in the family of solutions depicted in Fig. 8, where oscillatory behavior 
occurs for curves that reach very low, nonzero values in $V(t)$. When considering the biological relevance of the model, those curves should be discarded, since extremely low values of $V(t)$ correspond in practice to eradication. This is a result of the threshold we assume for the complete depletion of the cancer mass, when we postulate that if $V\left(t^{*}\right)=0$ with $\frac{d V}{d t}\left(t^{*}\right) \leq 0$, then for all subsequent times $t>t^{*}, V(t)=0$. The condition can be relaxed without altering the analytical results presented or changing the model equations, for example choosing a higher threshold which is not strictly zero and corresponds to the volume of half a cell.

Secondly, the model allows for an infinite supply of T-cells and for the tumour volume to grow without bound. Neither of these assumptions is strictly valid, and in a clinical application the model breaks down when tumour size exceeds the limits discussed in Sec. 2 or when the T-cells supply exceeds a biologically feasible size. Due to the diffusion limit of oxygen, the model is in fact valid for small tumours of the order of one cubic millimeter.

Analysis of our system shows that there is a maximum size for tumours that can oscillate perpetually. A further prediction of the model, when applied without the caveats, is that if tumours grow beyond this size, cancers get eliminated by an adequately strong response by T-cells. In other words, a larger tumour appears easier to find and kill, because, due to the model limitations discussed above, it elicits a stronger immune response and represents a bigger initial target, with a larger surface area to which T-cells can attach.

When taken without due care, this result may suggest that a delayed immune response that would allow a tumour to grow beyond the maximum threshold before initiating an immune attack could be more effective at counteracting tumours. Besides this result being a consequence of the assumptions used in the model, it should be clear that such a proposition is very risky. In fact, if a tumour were allowed to grow too long in the absence of an immune response, it may develop into a more aggressive malignancy or metastasize quickly with detrimental consequences for the patient's health. Also, given that there is relevant evidence that the immune system is capable of eliminating small tumours, pharmacologically enacting a delayed response by the innate immune surveillance may be unfeasible or have disastrous effects. On the other hand, it is important to observe that latent tumours may not become malignant, since it may be the immunoediting process that in fact hastens the transition to a more malignant tumour [49]. So, to sum up, although this solution is mathematically interesting and sheds light on the outcome of an immune response under the hypotheses of our model, suggesting viable therapeutic strategies with the aid of Eqs. (1)-(2) is beyond the scope of this paper. 
An additional point to note is that we have not considered possible further mutations in response to immune attack, so that, for example, long term oscillations in the system may change the susceptibility of cancers to immune attack. In some cancers, the growing tumour is immunoedited to remove recognized tumourassociated antigens so that by the time the disease is symptomatic the cytotoxic immune response is negligible and the cancer has progressed to a more aggressive state. We do not consider this case in our formulation.

A more practical and promising direction could be to identify parameters that lead to a reduced basin of attraction for perpetually oscillating solutions. A key factor affecting the size to which a tumour can grow without being eliminated is the surface area to volume ratio, captured by the shape factor $\rho$. As seen in Eq. (15), a larger $\rho$, corresponding to a tumour that is more accessible to CTLs, decreases the maximum size of tumour that can evade immune elimination. Hence, tumours whose geometries are more amenable to CTL-cancer cell contact are more likely to be eliminated without a cycle of regrowth. This result corroborates the observation that the presence of more tumour-infiltrating lymph nodes that can partially penetrate the tumour mass leads to a more favourable prognosis [31].

An advantage of the model presented here is that analytical solutions exist for all the three cases of typical dynamics. These solutions are interesting from a mathematical point of view and the nature of the problem allows for a semianalytical evaluation of periods of oscillations for certain classes of solutions. Specifically, it has been shown that the ratio $r_{\mathrm{t}} / k$ and the CTL killing rate $d_{\mathrm{c}}$ are important factors in regulating the frequency of oscillations. Periods tend to increase nonlinearly when CTLs have higher death rates and smaller killing rates. Also, as the ratio $r_{\mathrm{t}} / k$ gets closer to unity, oscillations become slower and slower, also resulting in nonlinear increases in amplitudes.

Unlike our equations, simulations occasionally present irregular oscillations, due to the probabilistic terms, which are absent in deterministic systems [6]. In the literature, there are also higher dimensional ODE models that give rise to a variety of oscillation types, with amplitudes either constant, increasing or decreasing with time [14]. Augmenting the dimensionality of the system generally increases the number of possible dynamical scenarios among cellular populations. For example, the model by Kirschner et al. [16] introduces a third differential equation for the mediation of natural and specific immunity by proteine hormones such as cytokines. This generates more outcomes for the tumour-CTLs oscillations.

The birth of self-sustained oscillations in higher dimensional models is due to the presence of a Hopf point [50] and not to the existence of a centre, which is in- 
stead a bifurcation that is not mentioned in any of the low dimensional ODEs currently available in the literature. Although for the typical class of two-dimensional systems for immunotherapies limit cycles cannot be theoretically ruled out [13], we are not aware of any work in the literature that reports self-sustained, stable oscillations when a constant (or null) influx of lymphocytes is assumed. Oscillations have instead been forced onto the system by using external terms in the equations, typically representing non stationary influxes of T-cells [43, 51]. These terms mimic the so-called periodic therapies that are clinically employed for treating cancer patients.

In a different context, two-dimensional and higher order models have also been employed for the mathematical description of tumour dormancy $[52,53]$, which is a condition where tumour cells persists in a host without growing. Experimental results have shown that some tumours can counteract an effective immune response, remain at low concentration in the system and regrow years later [54]. The modified equations discussed in Appendix B seem to capture some details of this phenomenon.

It will be interesting in future work to relax some of the model assumptions presented here and investigate how the solutions discussed in this paper are affected. For example, the hypothesis of a limitless supply of T-cells could be dropped and the model equations could be augmented with a term that bounds the total concentration of leukocytes in the system or that limits the influx of Tcells from lymph nodes to a given amount at any time. It is likely that this term will also alter the geometry of solutions and change the character and/or the stability of equilibria in the phase space. A future development of the model will also be to introduce stochastic effects to account for the degree of randomness inherently associated with the process of CTL killing of tumour cells. Furthermore, Eqs. (1)-(2) allow for a natural extension to include alternative tumour growth rates, such as Gompertzian, von Bertalanffy, logistic, etc. $[13,55]$. The next step in this analysis will be to understand how these and other factors can change the character of the solutions and how they impact the efficacy of the anti-tumour

response. Investigating the effect of a periodic influx of T-cells can also prove beneficial from the clinical point of view.

\section{Acknowledgments}

The work of PSK was supported by an ARC Discovery Early Career Research Award. The work of FF, BDH and KAL was supported by an ARC Discovery 
Project. The authors thank an anonymous reviewer for the constructive and stimulating comments, which helped to improve the quality of this paper significantly.

\section{References}

[1] R. Jaini, P. Kesaraju, J. M. Johnson, C. Z. Altuntas, D. Jane-Wit, V. K. Tuohy, An autoimmune-mediated strategy for prophylactic breast cancer vaccination, Nat. Med. 16 (2010) 799-803.

[2] R. Soiffer, F. S. Hodi, F. Haluska, K. Jung, S. Gillessen, S. Singer, K. Tanabe, R. Duda, S. Mentzer, M. Jaklitsch, R. Bueno, S. Clift, S. Hardy, D. Neuberg, R. Mulligan, I. Webb, M. Mihm, G. Dranoff, Vaccination with irradiated, autologous melanoma cells engineered to secrete granulocyte-macrophage colony-stimulating factor by adenoviral-mediated gene transfer augments antitumor immunity in patients with metastatic melanoma, J. Clin. Oncol. 21 (2003) 3343-3350.

[3] R. Soiffer, T. Lynch, M. Mihm, K. Jung, C. Rhuda, J. C. Schmollinger, F. S. Hodi, L. Liebster, P. Lam, S. Mentzer, S. Singer, K. K. Tanabe, A. B. Cosimi, R. Duda, A. Sober, A. Bhan, J. Daley, D. Neuberg, G. Parry, J. Rokovich, L. Richards, J. Drayer, A. Berns, S. Clift, L. K. Cohen, R. C. Mulligan, G. Dranoff, Vaccination with irradiated autologous melanoma cells engineered to secrete human granulocyte-macrophage colony-stimulating factor generates potent antitumor immunity in patients with metastatic melanoma, Proc. Natl. Acad. Sci. U.S.A. 95 (1998) 13141-13146.

[4] W. Wang, J. Epler, L. G. Salazar, S. R. Riddell, Recognition of breast cancer cells by CD8+ cytotoxic T-cell clones specific for NY-BR-1, Cancer Res. 66 (2006) 6826-6833.

[5] F. O. Nestle, G. Tonel, A. Farkas, Cancer vaccines: the next generation of tools to monitor the anticancer immune response, PLoS Med. 2 (2005) e339.

[6] P. Kim, P. Lee, Modeling protective anti-tumor immunity via preventative cancer vaccines using a hybrid agent-based and delay differential equation approach, PLoS Comp Bio 8 (10).

[7] B. J. Kennedy, Cyclic leukocyte oscillations in chronic myelogenous leukemia during hydroxyurea therapy, Blood 35 (1970) 751-760. 
[8] H. Vodopick, E. Rupp, C. Edwards, F. Goswitz, J. Beauchamp, Spontaneous cyclic leukocytosis and thrombocytosis in chronic granulocytic leukemia, New Engl. J. Med. 286 (6) (1972) 284-290.

[9] B. Mehta, M. Agarwal, Cyclic oscillations in leukocyte count in chronic myeloid leukemia, Acta Haem. 63 (2) (1980) 68-70.

[10] A. Rodriguez, C. Lutcher, Marked cyclic leukocytosis leukopenia in chronic myelogenous leukemia, Am. J. Med. 60 (6) (1976) 1041-1047.

[11] G. Chikkappa, A. Chanana, P. Chandra, E. Cronkite, K. Thompson, Cyclic oscillation of blood neutrophils in a patient with multiple myeloma, Blood 55 (1) (1980) 61-66.

[12] J. Krikorian, C. Portlock, D. Cooney, S. Rosenberg, Spontaneous regression of non-hodgkin's lymphoma: A report of nine cases, Cancer 46 (9) (1980) 2093-2099.

[13] A. D’Onofrio, A general framework for modeling tumor-immune system competition and immunotherapy: Mathematical analysis and biomedical inferences, Physica D 208 (3-4) (2005) 220-235.

[14] R. Eftimie, J. L. Bramson, D. J. Earn, Interactions between the immune system and cancer: a brief review of non-spatial mathematical models, Bull. Math. Biol. 73 (1) (2011) 2-32.

[15] L. G. de Pillis, A. E. Radunskaya, C. L. Wiseman, A validated mathematical model of cell-mediated immune response to tumor growth, Cancer Res. 65 (17) (2005) 7950-7958.

[16] D. Kirschner, J. C. Panetta, Modeling immunotherapy of the tumor-immune interaction, J Math Biol 37 (1998) 235-252.

[17] V. A. Kuznetsov, I. A. Makalkin, M. A. Taylor, A. S. Perelson, Nonlinear dynamics of immunogenic tumors: parameter estimation and global bifurcation analysis, Bull. Math. Biol. 56 (2) (1994) 295-321.

[18] K. León, A. Lage, J. Carneiro, How regulatory CD25+CD4+ T cells impinge on tumor immunobiology: the differential response of tumors to therapies, $\mathrm{J}$ Immunol. 179 (9) (2007) 5659-5668. 
[19] K. León, A. Lage, J. Carneiro, How regulatory CD25+CD4+ T cells impinge on tumor immunobiology? on the existence of two alternative dynamical classes of tumors, J Theor Biol. 247 (1) (2007) 122-137.

[20] A. Lin, A model of tumor and lymphocyte interactions, Discrete and Continuous Dynamical Systems - Series B 4 (1) (2004) 241-266.

[21] S. J. Merrill, A model of the role of natural killer cells in immune surveillance - I, J Math Biol. 12 (1981) 363-373.

[22] H. Moore, N. K. Li, A mathematical model for chronic myelogenous leukemia (CML) and T cell interaction, J Theor Biol. 225 (4) (2004) 513523.

[23] A. Matzavinos, M. A. Chaplain, V. A. Kuznetsov, Mathematical modelling of the spatio-temporal response of cytotoxic T-lymphocytes to a solid tumour, Math Med Biol 21 (1) (2004) 1-34.

[24] N. Bellomo, N. K. Li, P. K. Maini, On the foundations of cancer modelling: selected topics, speculations, and perspectives, Mathematical Models and Methods in Applied Sciences 18 (4) (2008) 593-646.

[25] T. Roose, S. J. Chapman, P. K. Maini, Mathematical models of avascular tumor growth, SIAM Review 49 (2) (2007) 179-208.

[26] A. R. Kansal, S. Torquato, I. V. Harsh GR, E. A. Chiocca, T. S. Deisboeck, Simulated brain tumor growth dynamics using a three-dimensional cellular automaton, J. Theor. Biol. 203 (4) (2000) 367-382.

[27] A. A. Patel, E. T. Gawlinski, S. K. Lemieux, R. A. Gatenby, A cellular automaton model of early tumor growth and invasion, J. Theor. Biol. 213 (3) (2001) 315-331.

[28] T. Alarcón, H. M. Byrne, P. K. Maini, A cellular automaton model for tumour growth in inhomogeneous environment, J. Theor. Biol. 225 (2003) $257-274$.

[29] A. R. Anderson, M. A. Chaplain, Continuous and discrete mathematical models of tumor-induced angiogenesis, Bull. Math. Biol. 60 (5) (1998) 857899. 
[30] L. G. de Pillis, D. G. Mallet, A. E. Radunskaya, Spatial tumor-immune modeling, Computational and Mathematical Methods in Medicine 7 (2-3) (2006) 159-176.

[31] D. G. Mallet, L. G. De Pillis, A cellular automata model of tumor-immune system interactions, J. Theor. Biol. 239 (2006) 334-350.

[32] A. S. Qi, X. Zheng, C. Y. Du, B. S. An, A cellular automaton model of cancerous growth, J. Theor. Biol. 161 (1) (1993) 1-12.

[33] C. R. Mackay, Chemokine receptors and T cell chemotaxis, J. Exp. Med. 184 (3) (1996) 799-802.

[34] M. Maurer, E. von Stebut, Macrophage inflammatory protein-1, Int. J. Biochem. Cell Biol. 36 (10) (2004) 1882-1886.

[35] A. Brú, S. Albertos, J. Subiza, J. Garca-Asenjo, I. Brú, The universal dynamics of tumor growth, Biophys. J. 85 (5) (2003) 2948-2961.

[36] I. Van Leeuwen, C. Zonneveld, From exposure to effect: A comparison of modeling approaches to chemical carcinogenesis, Rev. Mutat. Res. 489 (1) (2001) 17-45.

[37] D. M. Catron, A. A. Itano, K. A. Pape, D. L. Mueller, M. K. Jenkins, Visualizing the first $50 \mathrm{hr}$ of the primary immune response to a soluble antigen, Immunity 21 (3) (2004) 341-347.

[38] T. Kuroishi, S. Tominaga, T. Morimoto, H. Tashiro, S. Itoh, H. Watanabe, M. Fukuda, J. Ota, T. Horino, T. Ishida, Tumor growth rate and prognosis of breast cancer mainly detected by mass screening, Jpn. J. Cancer Res. 81 (5) (1990) 454-462.

[39] J. Michaelson, S. Satija, R. Moore, G. Weber, E. Halpern, A. Garland, D. B. Kopans, Estimates of breast cancer growth rate and sojourn time from screening database information, Journal of Women's Imaging 5 (1) (2003) $11-19$.

[40] J. A. Spratt, D. von Fournier, J. S. Spratt, E. E. Weber, Decelerating growth and human breast cancer, Cancer 71 (1993) 2013-2019. 
[41] H. Weedon-Fekjaer, B. H. Lindqvist, L. J. Vatten, O. O. Aalen, S. Tretli, Breast cancer tumor growth estimated through mammography screening data, Breast Cancer Res. 10 (2008) R41.

[42] R. J. De Boer, D. Homann, A. S. Perelson, Different dynamics of CD4+ and $\mathrm{CD} 8+\mathrm{T}$ cell responses during and after acute lymphocytic choriomeningitis virus infection, J Immunol. 171 (8) (2003) 3928-3935.

[43] O. Sotolongo-Costa, L. Molina, D. Perez, J. Antoranz, M. Reyes, Behavior of tumors under nonstationary therapy, Phys. D 178 (3-4) (2003) 242-253.

[44] S. H. Strogatz, Nonlinear dynamics and chaos, Westview Press, Cambridge, MA, 2001.

[45] R. Corless, G. Gonnet, D. Hare, D. Jeffrey, D. Knuth, On the Lambert W function, Adv Comp Math 5 (4) (1996) 329-359.

[46] R. Haberman, Mathematical Models: Mechanical Vibrations, Population Dynamics, and Traffic Flow, Prentice-Hall, Englewood Cliffs, NJ, 1977.

[47] U. Foryś, J. Waniewski, P. Zhivkov, Anti-tumor immunity and tumor antiimmunity in a mathematical model of tumor immunotherapy, J. Biol. Syst. 14 (1) (2006) 13-30.

[48] H. De Vladar, J. Gonzlez, Dynamic response of cancer under the influence of immunological activity and therapy, J. Theor. Biol. 227 (3) (2004) 335-348.

[49] G. Dunn, A. Bruce, H. Ikeda, L. Old, R. Schreiber, Cancer immunoediting: From immunosurveillance to tumor escape, Nature Immunol. 3 (11) (2002) 991-998.

[50] Y. A. Kuznetsov, Elements of Applied Bifurcation Theory, Springer-Verlag, New York, NY, 2004.

[51] A. D'Onofrio, A. Gandolfi, Tumour eradication by antiangiogenic therapy: Analysis and extensions of the model by hahnfeldt et al. (1999), Math. Bio. 191 (2) (2004) 159-184.

[52] K. P. Wilkie, A review of mathematical models of cancer-immune interaction in the context of tumor dormancy, in: H. Enderling, N. Almog, L. Hlatky (Eds.), System Biology of Tumor Dormancy, Springer, New York, NY, 2013, Ch. 10, pp. 201-234. 
[53] K. Wilkie, P. Hahnfeldt, Mathematical models of immune-induced cancer dormancy and the emergence of immune evasion, Interface Focus 3 (4).

[54] A. Saudemont, B. Quesnel, In a model of tumor dormancy, long-term persistent leukemic cells have increased b7-h1 and b7.1 expression and resist CTL-mediated lysis, Blood 104 (7) (2004) 2124-2133.

[55] M. Marusic, Z. Bajzer, J. Freyer, S. Vuk-Pavlovic, Analysis of growth of multicellular tumour spheroids by mathematical models, Cell Prolif. 27 (2) (1994) 73-94.

[56] H. T. Davis, Introduction to nonlinear differential and integral equations, Dover Publications, New York, NY, 1962. 
Table 1: Typical ranges and units of measurement for the variables and parameters used in the model.

\begin{tabular}{llll}
\hline Symbol & Meaning & Typical ranges & Unit of measurement \\
\hline$V(t)$ & tumour volume & {$\left[0-4 \times 10^{6}\right]$} & $\mu \mathrm{m}^{3}$ \\
$C(t)$ & CTL (dimensionless) concentration or coverage & {$[0-20]$} & none \\
\hline$\rho$ & shape factor & {$[36 \pi)^{1 / 3}$} & none \\
$r_{\mathrm{t}}$ & tumour growth rate & {$[0.0-0.5]$} & $\mu \mathrm{m} \cdot \mathrm{day}^{-1}$ \\
$k$ & CTL killing rate of tumour cells & {$[0.0-0.5]$} & $\mu \mathrm{m} \cdot \mathrm{day}^{-1}$ \\
$r_{\mathrm{c}}$ & CTL recruitment rate & {$[0.0-0.2]$} & $\mu \mathrm{m}^{-2} \cdot \mathrm{day}^{-1}$ \\
$d_{\mathrm{c}}$ & CTL death rate & {$[0.0-0.5]$} & day $^{-1}$ \\
\hline
\end{tabular}

(a)
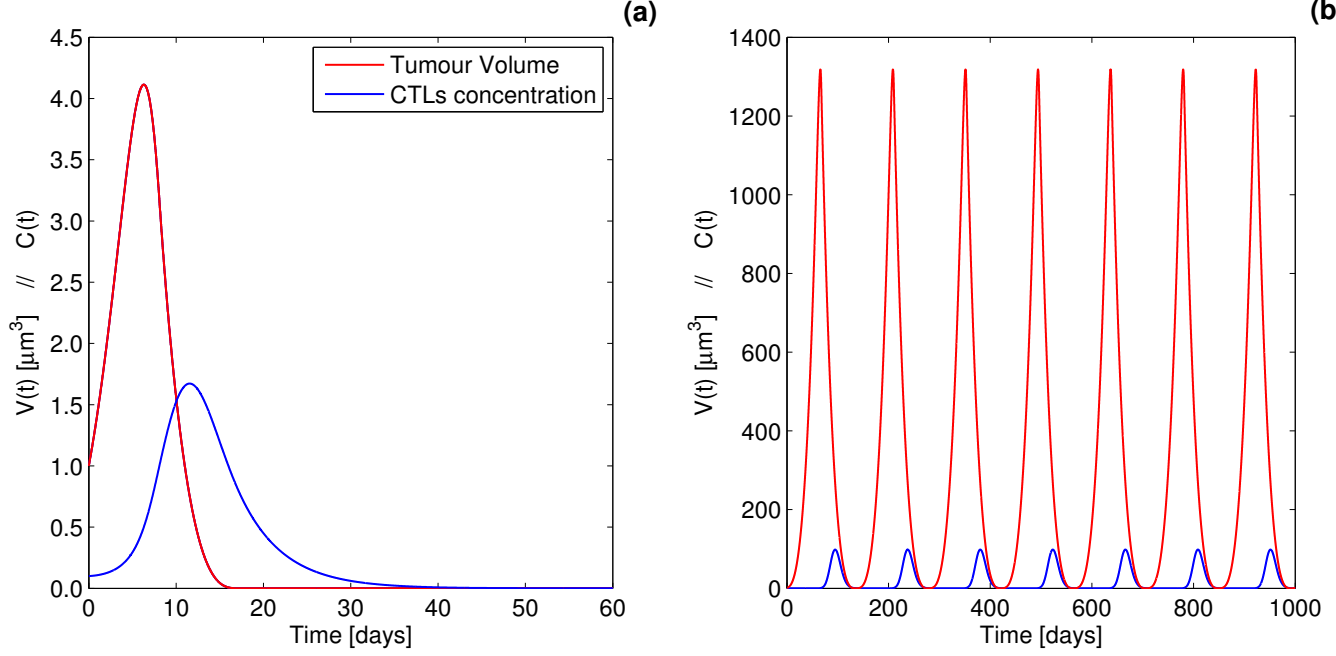

Figure 1: Example of two possible types of solutions of the model. In (a), the tumour is eradicated in finite time. The solution for $V(t)$ reaches zero approximately at time $t=15$ days. In (b), the populations of CTLs and tumour cells oscillate, and the cancer is never destroyed. Parameters for the two scenarios have common values $r_{\mathrm{t}}=0.1 \mu \mathrm{m} \cdot \mathrm{day}^{-1}, k=0.2 \mu \mathrm{m} \cdot \mathrm{day}^{-1}, d_{\mathrm{c}}=0.2 \mathrm{day}^{-1}$, but recruitment rates are different. For (a): $r_{\mathrm{c}}=0.05 \mu \mathrm{m}^{-2} \cdot$ day $^{-1}$, for (b): $r_{\mathrm{c}}=0.001 \mu \mathrm{m}^{-2}$. day $^{-1}$. Note the different maxima for $V(t)$ and $C(t)$ and the different temporal scales in (a) and (b). 


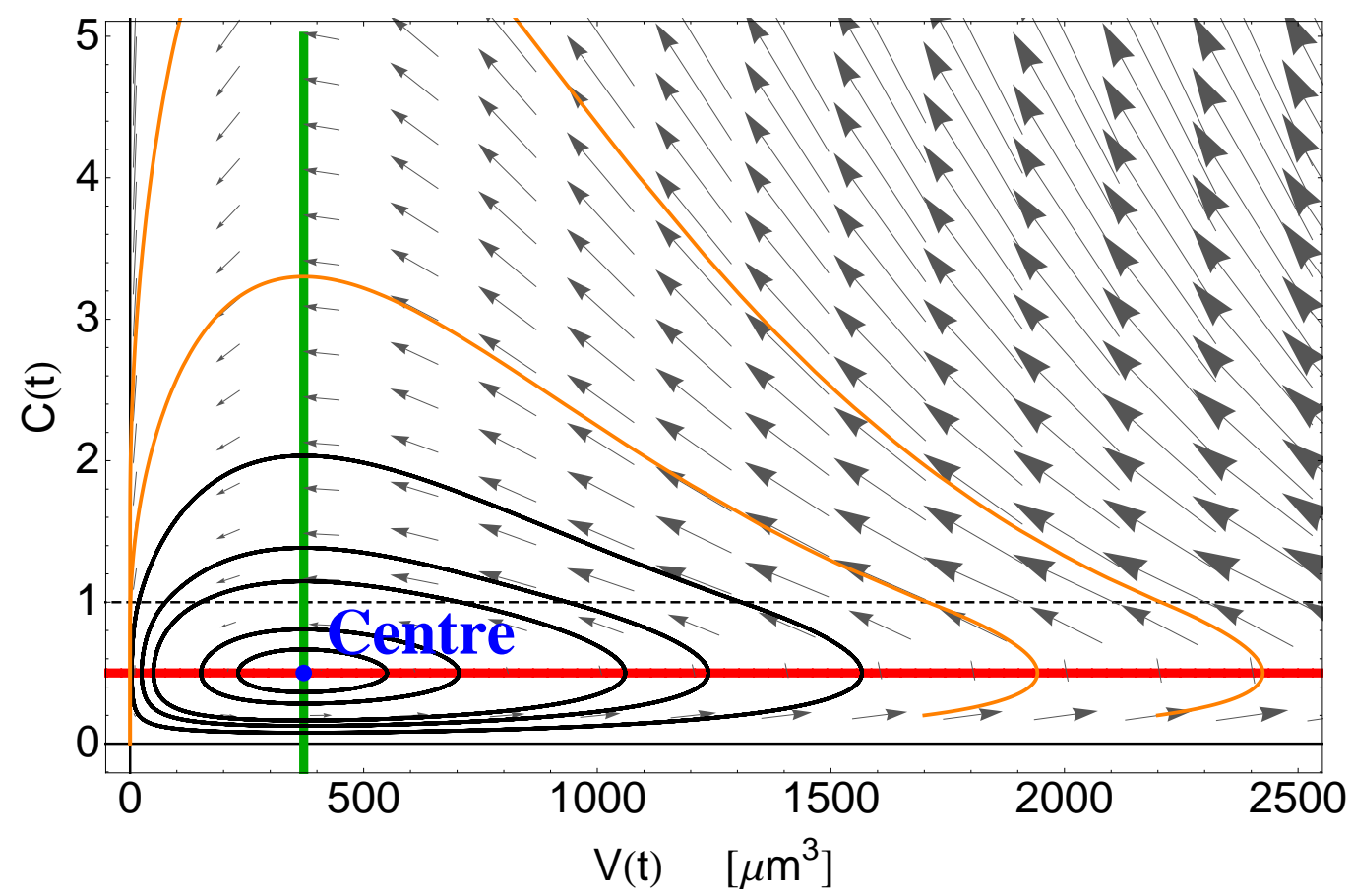

Figure 2: A centre is present in the $(V, C)$-plane if $r_{\mathrm{t}}<k$ at the intersection of the two relevant nullclines indicated in red and green. The coordinates of this centre are given by $\left(V_{\odot}, C_{\odot}\right)=$ $\left(\left(d_{\mathrm{c}} /\left(\rho r_{\mathrm{c}}\right)\right)^{3 / 2}, r_{\mathrm{t}} / k\right)$. Arrows indicate the intensity of the flow field associated with Eqs. (1)-(2). In this scenario, the tumour volume $V(t)$ can either periodically regrow or be eradicated in finite time. Oscillatory solutions in the $(V, C)$-plane are shown in black, and solutions leading to tumour eradication are shown in orange. The dashed line delimits the boundary between the regions $C(t) \geq 1$ and $0<C(t)<1$, relevant to the term $\min \{1, C(t)\}$ in Eq. (1). Note that model equations also admit the nullclines $V=0$ and $C=0$, which are not plotted since they are irrelevant for the dynamics. 


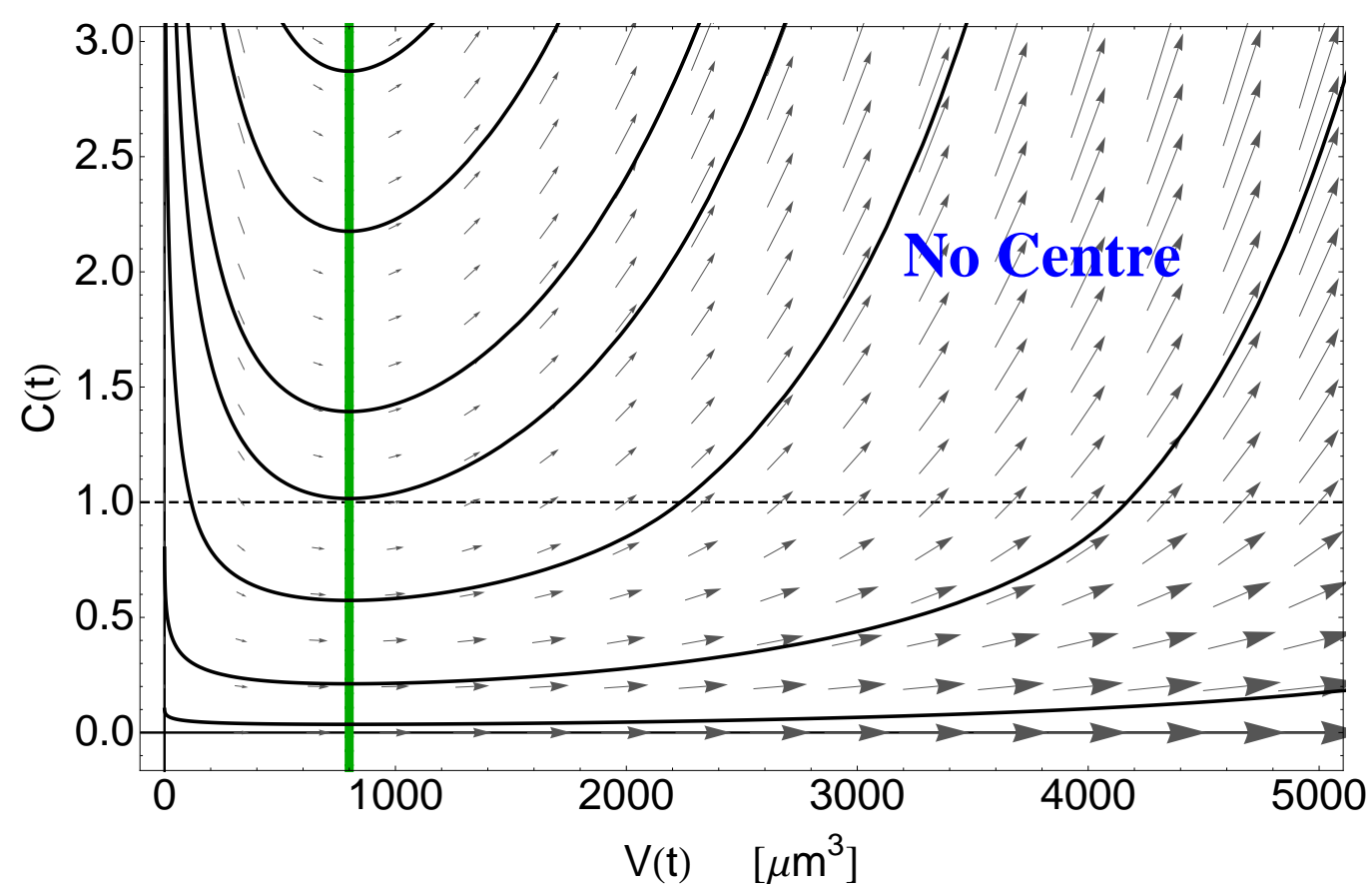

Figure 3: If $r_{\mathrm{t}}>k$, only one relevant nullcline is present in the $(V, C)$-plane and solutions are always unbounded. Note that the other existing nullclines $V=0$ and $C=0$ are not plotted. 
(a)

(b)
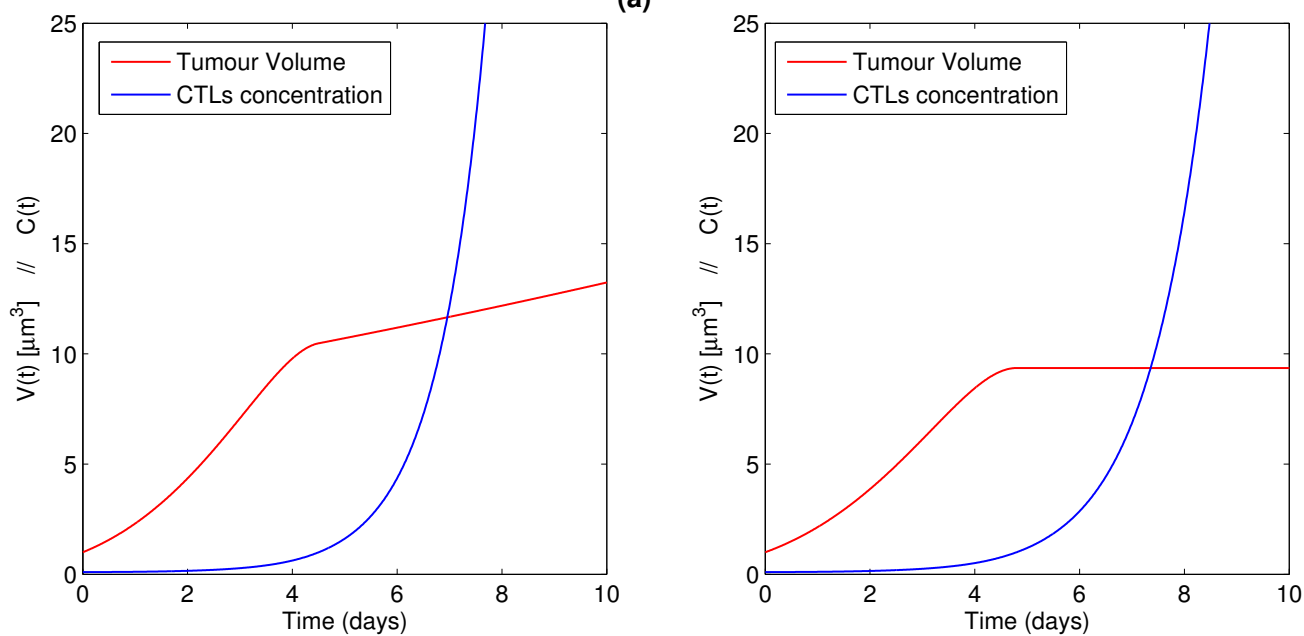

Figure 4: Example of typical time evolutions for the unbounded and the degenerate cases in (a) and (b) respectively. Parameters are given by $k=0.2 \mu \mathrm{m} \cdot$ day $^{-1}, d_{\mathrm{c}}=0.2$ day $^{-1}, r_{\mathrm{c}}=0.05 \mu \mathrm{m}^{-2}$ $\cdot$ day $^{-1}$ and $r_{\mathrm{t}}=0.22 \mu \mathrm{m} \cdot$ day $^{-1}$ for (a) and $r_{\mathrm{t}}=0.2 \mu \mathrm{m} \cdot$ day $^{-1}$ for (b). Note that $V(t)$ changes its rate of growth in (a) when $C(t)=1$ and that it reaches a plateau in (b). The tumour volume then increases or remains at a constant value whilst the concentration of CTLs grows without bound. 


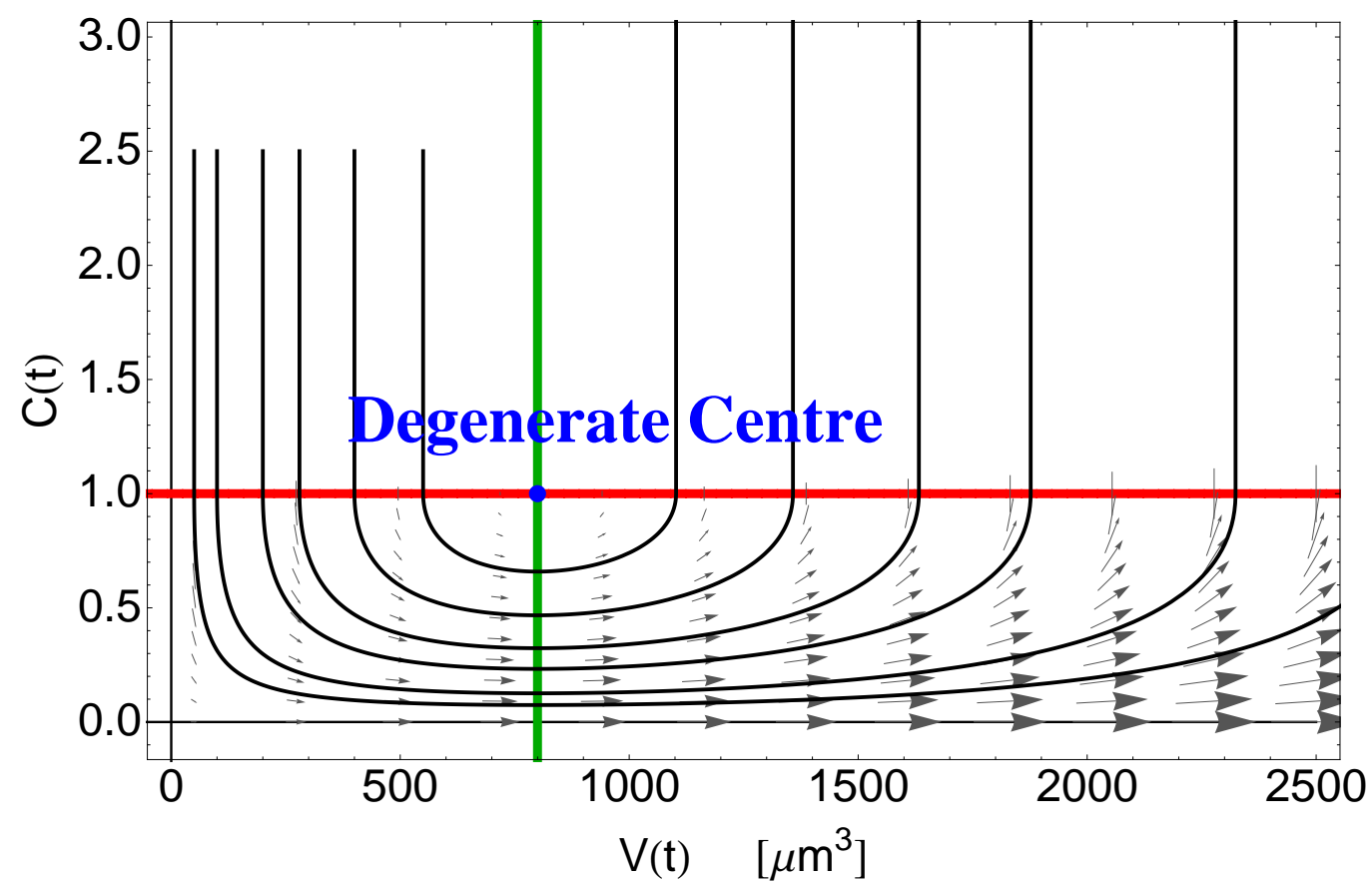

Figure 5: If $r_{\mathrm{t}}=k$, two relevant nullclines exist in the $(V, C)$-plane, where the second nullcline is now $C=1$. This situation is labelled as degenerate due the fact that in the whole half-plane $C \geq 1$, one eigenvalue of the Jacobian is always zero. Flowlines for $C \geq 1$ are vertical lines (not shown to avoid clutter). Consequently, solutions perform a half turn below the degenerate centre and then increase vertically towards infinity, because, for $C \geq 1, V(t)=$ constant, while $C(t) \rightarrow \infty$ as $t \rightarrow \infty$. 

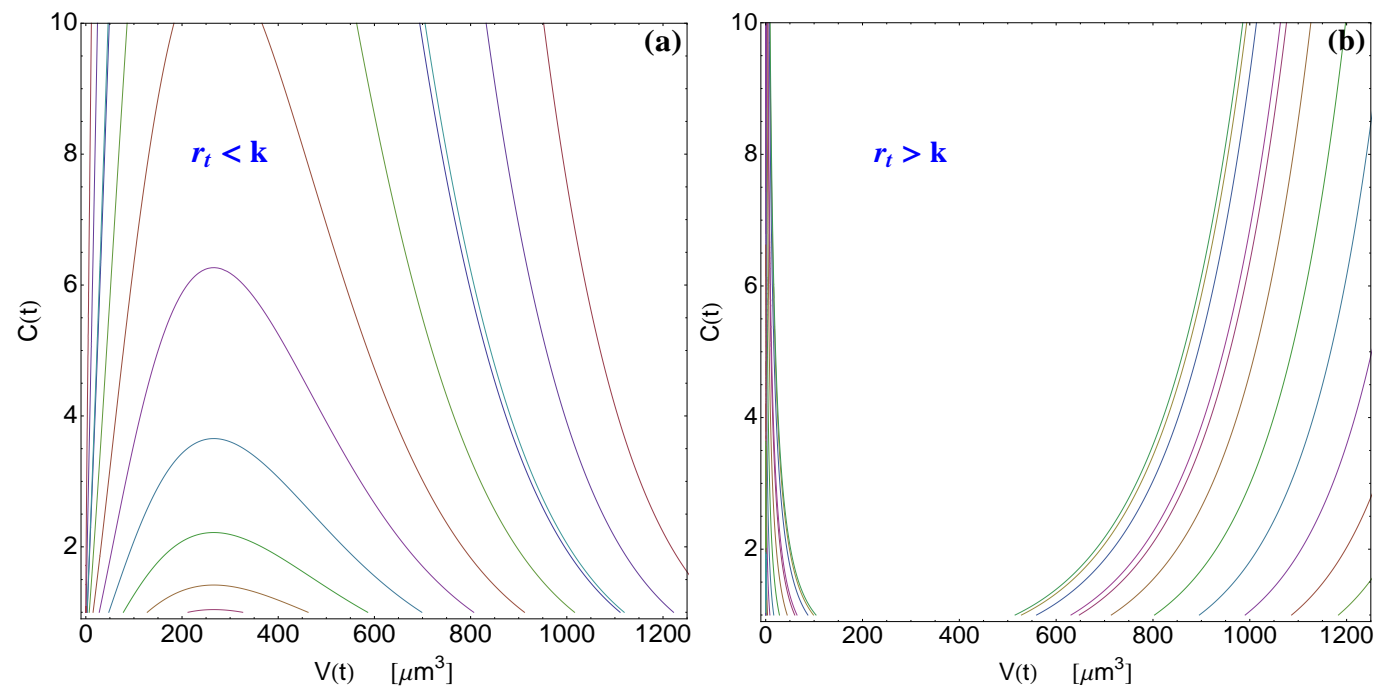

Figure 6: Examples of solutions from Eqs. (11)-(12) with different initial conditions $\left(V_{I}, 1\right)$ in the half-plane $C(t) \geq 1$, for (a) the bounded $\left(r_{\mathrm{t}}<k\right)$ and (b) the unbounded $\left(r_{\mathrm{t}}>k\right)$ cases. Parameters are given by $k=0.2 \mu \mathrm{m} \cdot$ day $^{-1}, d_{\mathrm{c}}=0.2$ day $^{-1}, r_{\mathrm{c}}=0.001 \mu \mathrm{m}^{-2} \cdot$ day $^{-1}$, and $r_{\mathrm{t}}=0.1 \mu \mathrm{m}$. day $^{-1}$ for (a) and $r_{\mathrm{t}}=0.3 \mu \mathrm{m} \cdot$ day $^{-1}$ for (b). Note that the maxima occurring in (a) are at the $V$ value corresponding to the green nullcline in Fig. 2, i.e. $V_{\odot}=\left(d_{\mathrm{c}} /\left(\rho r_{\mathrm{c}}\right)\right)^{3 / 2}$. 

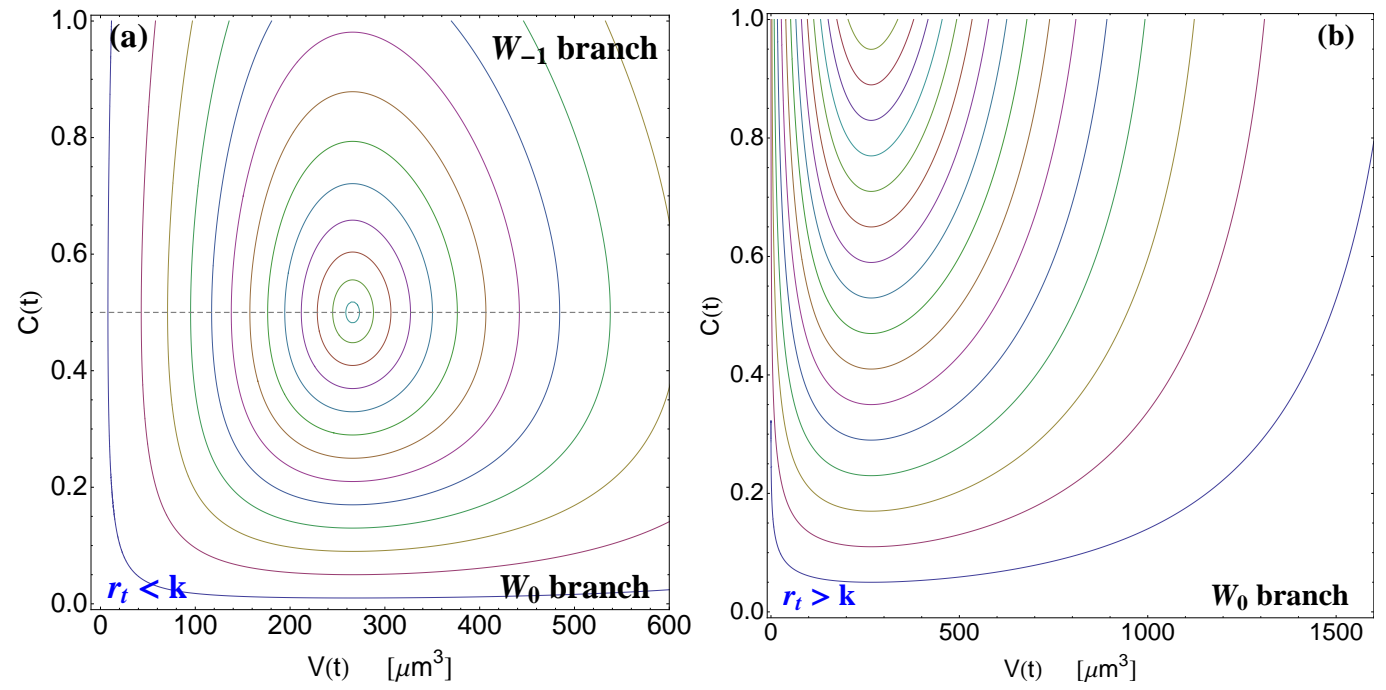

Figure 7: Solutions in the region $0<C(t)<1$ for (a) bounded $\left(r_{\mathrm{t}}<k\right)$ and (b) unbounded $\left(r_{\mathrm{t}}>k\right)$ scenarios and for different initial conditions $\left(V_{0}, C_{0}\right)$. Parameters are given by: $k=0.2 \mu \mathrm{m}$. day $^{-1}, d_{\mathrm{c}}=0.2$ day $^{-1}, r_{\mathrm{c}}=0.001 \mu \mathrm{m}^{-2} \cdot$ day $^{-1}$, and $r_{\mathrm{t}}=0.1 \mu \mathrm{m} \cdot$ day $^{-1}$ for (a) and $r_{\mathrm{t}}=0.3$ $\mu \mathrm{m} \cdot$ day $^{-1}$ for (b). For case (a), $r_{\mathrm{t}} / k=1 / 2$, and each solution is described by the 0 branch of the Lambert $W$ function in Eq. (14) in the lower half of the region $0<C(t)<1$ and by the -1 branch in the upper half. For case (b), the 0 branch describes the solutions in the whole region. Note the different scales on the $V$-axis.
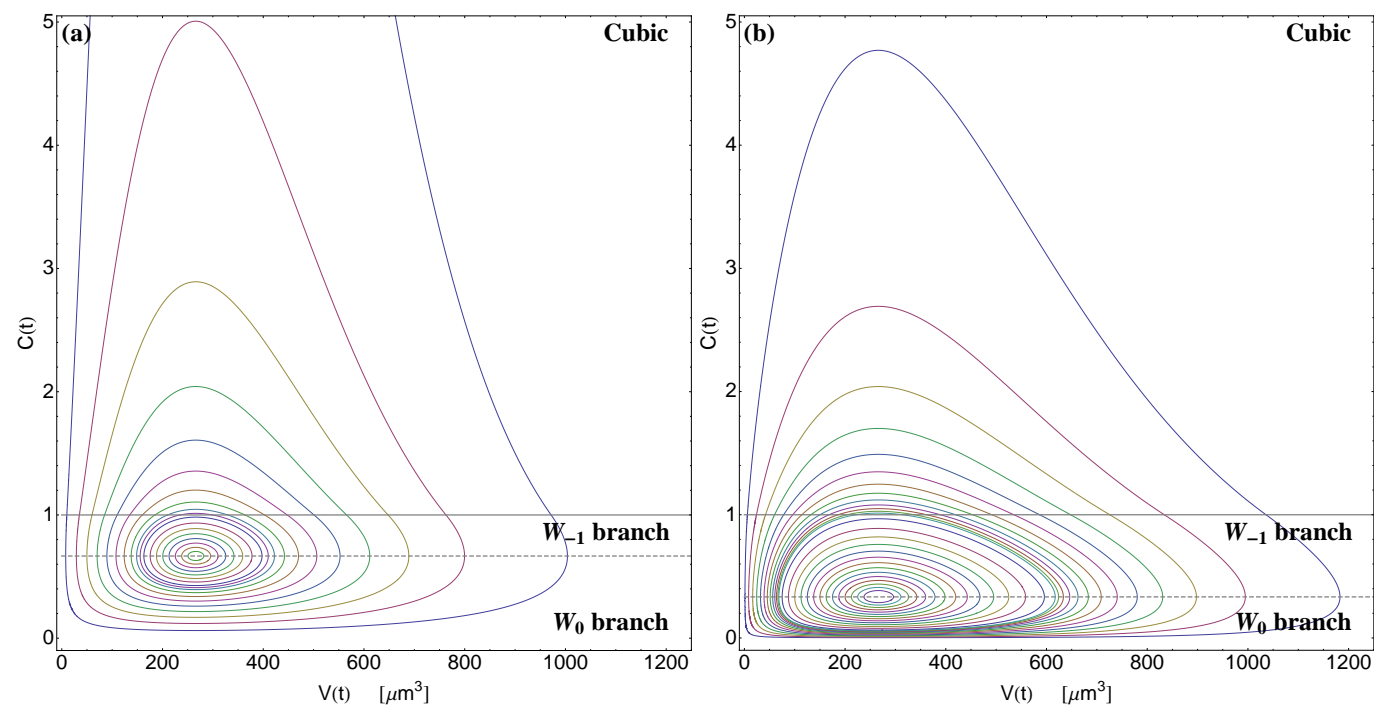

Figure 8: Example of complete solutions for the oscillatory case for $r_{\mathrm{t}} / k=2 / 3\left(r_{\mathrm{t}}=0.2 \mu \mathrm{m}\right.$. day $^{-1}$ and $k=0.3 \mu \mathrm{m} \cdot$ day $\left.^{-1}\right)$ in (a) and $r_{\mathrm{t}} / k=1 / 3\left(r_{\mathrm{t}}=0.1 \mu \mathrm{m} \cdot\right.$ day $^{-1}$ and $k=0.3 \mu \mathrm{m}$. day $^{-1}$ ) in (b). Other parameters have common values $d_{\mathrm{c}}=0.2 \mathrm{day}^{-1}, r_{\mathrm{c}}=0.001 \mu \mathrm{m}^{-2} \cdot \mathrm{day}^{-1}$. 


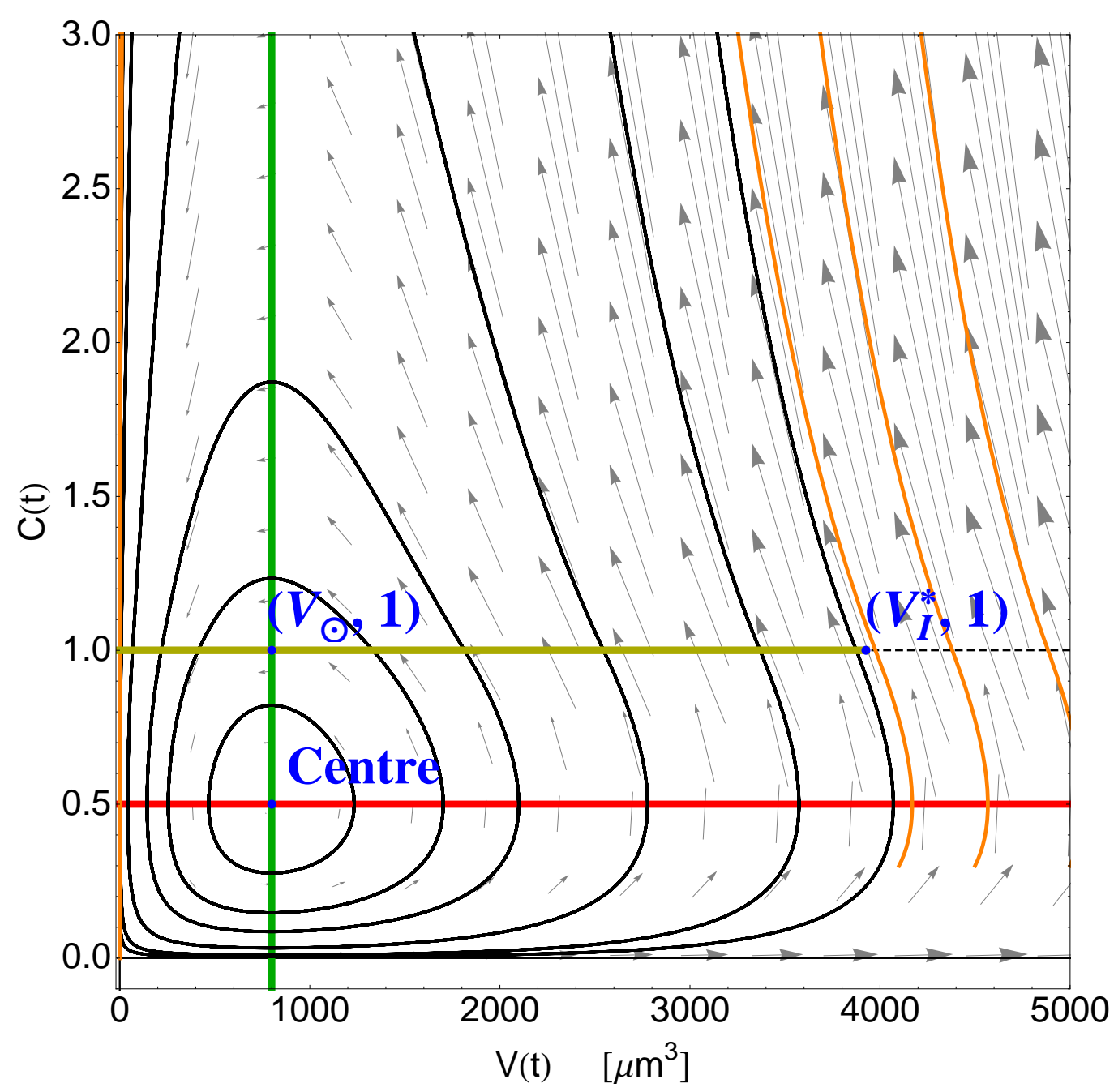

Figure 9: Basin of attraction for oscillatory orbits: orbits in black belong to the basin and orbits in orange represent tumours that are eradicated in a finite time. Orbits with a $V$-coordinate at $C=1$ that is smaller than $V_{I}^{*}=3 \sqrt{3} V_{\odot}$ are oscillatory. Relevant nullclines are in green and red, and the basin of attraction is in olive. 


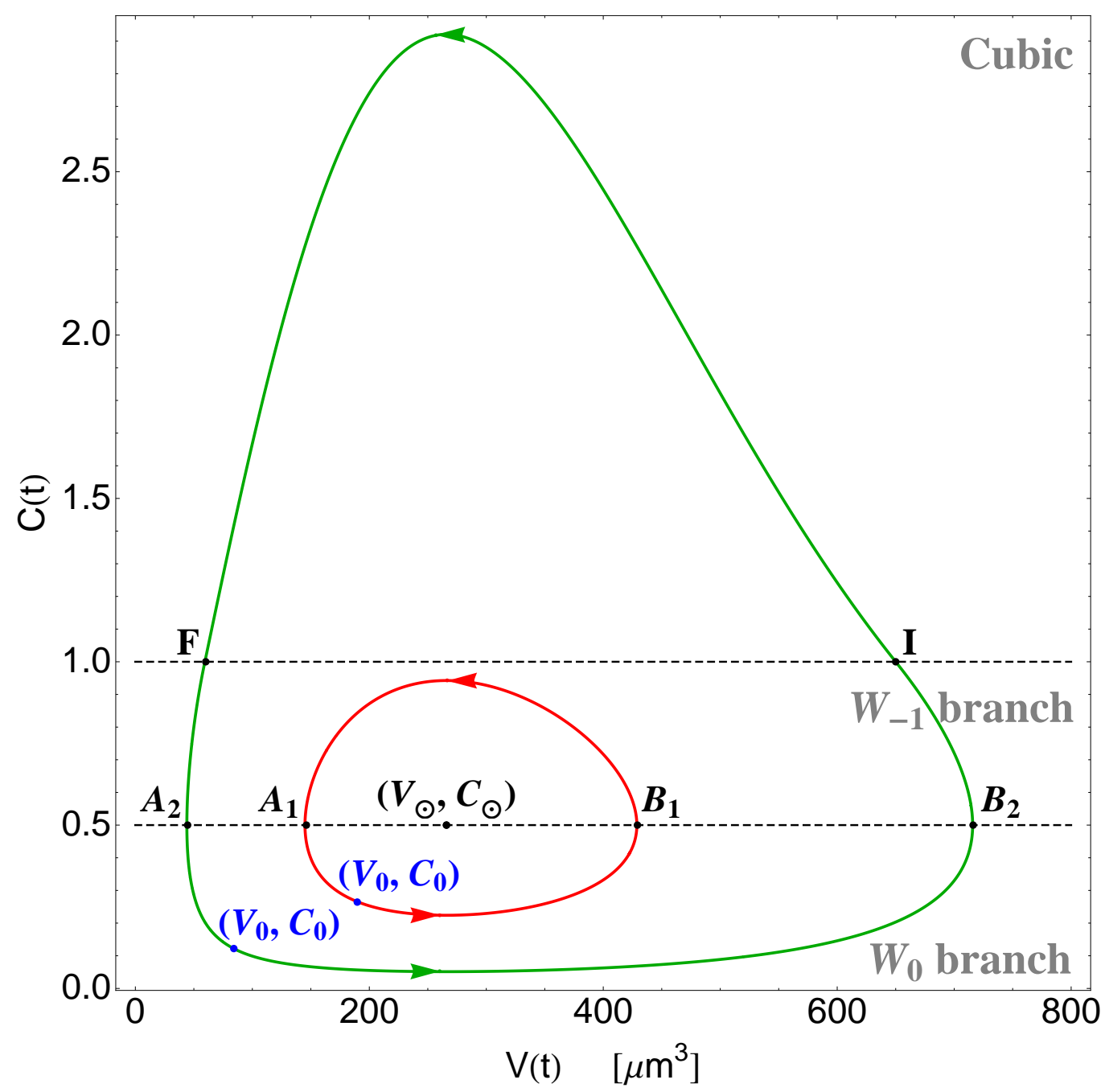

Figure 10: Examples of typical paths for the calculations of periods, for two bounded orbits when $r_{\mathrm{t}} / k=1 / 2$. The red, closed trajectory remains in the region $0<C(t)<1$, and its period is given by the sum of the two paths $A_{1} \rightarrow B_{1}$ and $B_{1} \rightarrow A_{1}$. This calculation involves only the two branches of the Lambert $W$ in Eq. (14). The green orbit exists also for $C(t) \geq 1$, and the calculation of the period for the section $I \rightarrow F$ is made using the cubic solution given by Eq. (11). These two orbits occur for the following model parameters: $d_{\mathrm{c}}=0.2 \mathrm{day}^{-1}, r_{\mathrm{c}}=0.001 \mu \mathrm{m}^{-2} \cdot$ day $^{-1}, k=0.2 \mu \mathrm{m}$ - day $^{-1}$ and $r_{\mathrm{t}}=0.1 \mu \mathrm{m} \cdot$ day $^{-1}$. Periods are $T \approx 86$ days and $T \approx 64$ days for the green and red cycles respectively. 


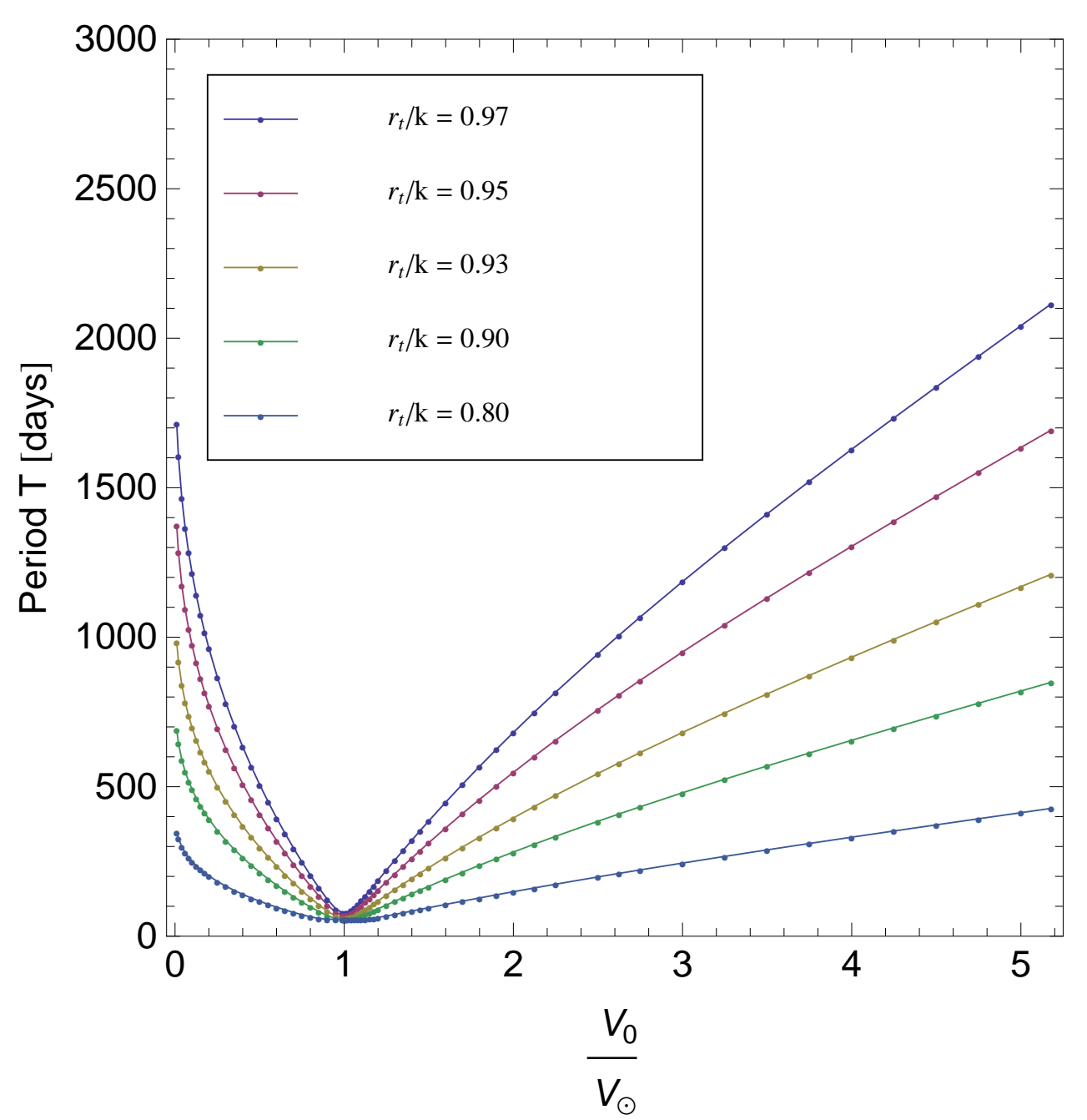

Figure 11: Numerical computation of periods of orbits for varying killing rates $k$ and the following values of other parameters: $d_{\mathrm{c}}=0.3 \mathrm{day}^{-1}, r_{\mathrm{c}}=0.001 \mu \mathrm{m}^{-2} \cdot$ day $^{-1}$, and $r_{\mathrm{t}}=0.1 \mu \mathrm{m} \cdot$ day $^{-1}$. Different initial conditions $V_{0}$ are chosen, whilst $C_{0}=9 / 10 C_{\odot}$ remains fixed. The ratio $V_{0} / V_{\odot}$ is on the abscissa, and the corresponding period for the given $r_{\mathrm{t}} / k$ is on the ordinate. Note that as the initial condition approaches the centre, i.e. as $V_{0} / V_{\odot} \rightarrow 1$, the period converges to the limiting value of the small oscillations, given by $2 \pi / \omega_{0}$ (see Eq. (8)). In this example the period is approximately 56.7 days. The way the period converges depends on the ratio $r_{\mathrm{t}} / k$, and the plot becomes steeper and more cusp-like as $r_{\mathrm{t}} / k$ approaches unity. For the limiting case $r_{\mathrm{t}} / k=1$, the solution is degenerate and diverges. Note also that for intersections occurring at $\left(V_{I}, 1\right)$ so that $V_{I} / V_{\odot}>3 \sqrt{3} \approx 5.196$, periodic solutions do not exist and the tumour is eradicated. 


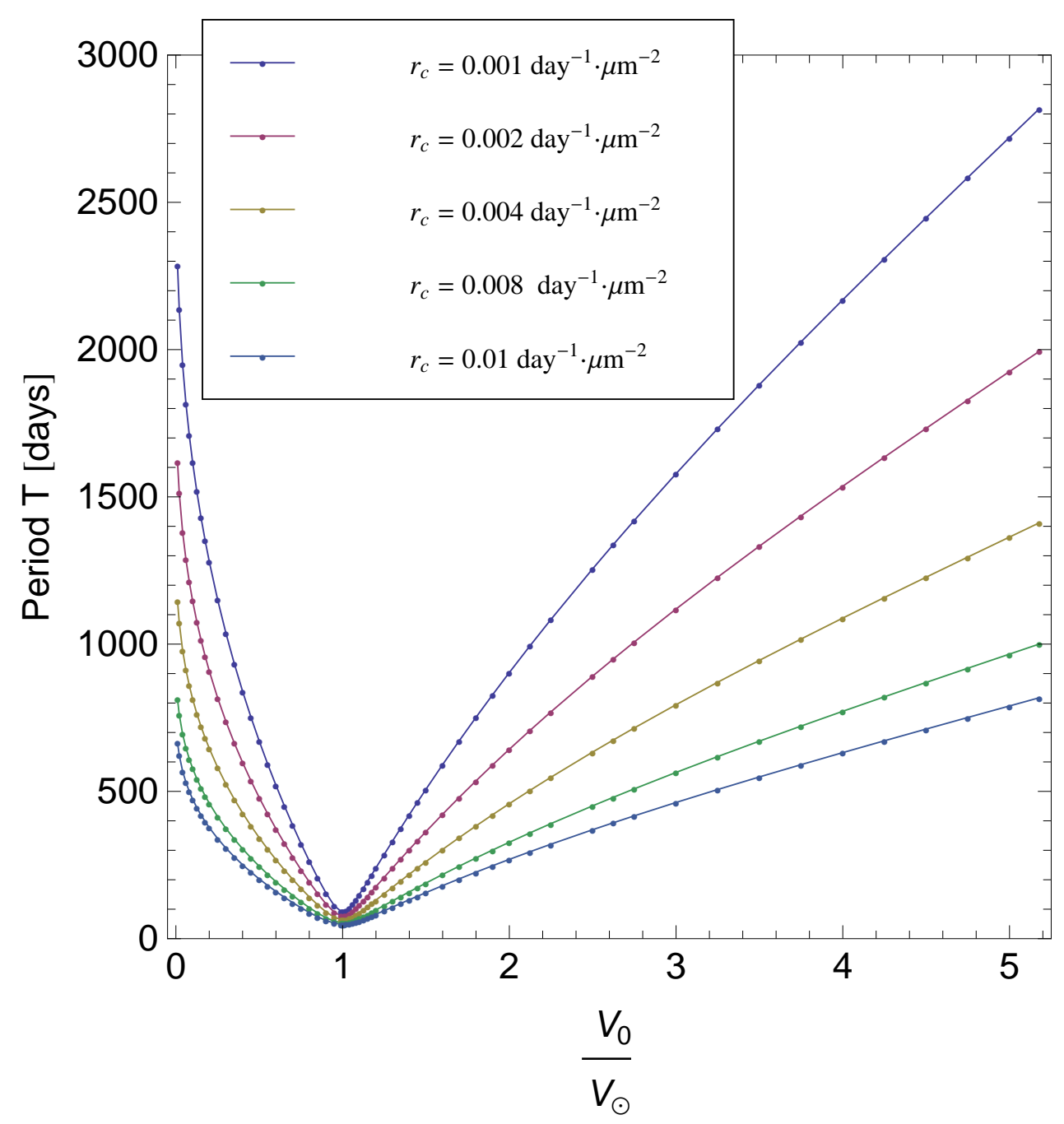

Figure 12: Numerical computation of periods of orbits for varying recruiting rates $r_{\mathrm{c}}$ for the following values of other parameters: $d_{\mathrm{c}}=0.3$ day $^{-1}, r_{\mathrm{t}}=0.1 \mu \mathrm{m} \cdot$ day $^{-1}$, and a ratio $r_{\mathrm{t}} / k=0.97$ (see Fig. 11). Similarly to the previous Figure, different $V_{0}$ are chosen, whilst $C_{0}=9 / 10 C_{\odot}$ is fixed. The ratio $V_{0} / V_{\odot}$ is on the abscissa, and the corresponding period for the given value of $r_{\mathrm{c}}$ is on the ordinate, with minima occurring in the small oscillations regime at $V_{0} / V_{\odot}=1$. Since the extension of the region of existence for these curves decreases as $r_{\mathrm{c}}$ increases, a value for the ratio $r_{\mathrm{t}} / k$ has been chosen so that the given range of $V_{0} / V_{\odot}$ is in the basin of attraction. Note that the minima occur at values of periods that increase as the rate of CTL recruitment, $r_{\mathrm{c}}$, decreases. The scale is the same as the one in Fig. 11. 


\section{Appendix A. Analytic solution of model equations when $C(t)<1$}

An analytic solution to the model equations when $C(t)<1$ can be found using the following strategy of substitutions. First, given the system

$$
\frac{d V}{d t}=\rho r_{\mathrm{t}} V^{2 / 3}-\rho k V(t)^{2 / 3} C, \quad \frac{d C}{d t}=\rho r_{\mathrm{c}} V^{2 / 3} C-d_{\mathrm{c}} C,
$$

we substitute $x(t)=V(t)^{1 / 3}$. Given that $V^{\prime}(t)=3 x^{2} x^{\prime}(t)$ and discarding the solution $x(t)=0$, we obtain the simplified equations

$$
\frac{d x}{d t}=\frac{1}{3} \rho\left(r_{\mathrm{t}}-k C\right), \quad \frac{d C}{d t}=\left(\rho r_{\mathrm{c}} x^{2}-d_{\mathrm{c}}\right) C .
$$

From this substitution, given that $x(t)$ is proportional to the radius of the spherical tumour, it is clear that the growth law is linear in the radius, as in Brú et al. [35]. From the first of these equations, an expression for $C(t)$ can be found, which can then be differentiated to obtain

$$
\frac{d C}{d t}=-\frac{3}{k \rho} \frac{d^{2} x}{d t^{2}}
$$

This expression can be substituted back into the second of Eqs (A.1), to obtain a second-order ordinary nonlinear differential equation that describes the system:

$$
\frac{d^{2} x}{d t^{2}}=\left(\rho r_{\mathrm{c}} x(t)^{2}-d_{\mathrm{c}}\right)\left[\frac{d x}{d t}-\frac{1}{3} \rho r_{\mathrm{t}}\right]
$$

It is trivial now to recast the system as two first-order equations, by defining $y(t)=$ $x^{\prime}(t)-\frac{1}{3} \rho r_{\mathrm{t}}$ to obtain

$$
\frac{d x}{d t}=y+\frac{1}{3} \rho r_{\mathrm{t}}, \quad \frac{d y}{d t}=y\left(\rho r_{\mathrm{c}} x^{2}-d_{\mathrm{c}}\right) .
$$

These equations are generalisations of the well-known Lotka-Volterra equations, with a third-order term in the equation for $y(t)$ [56]. Solutions for $x=x(t)$ and $y=y(t)$ exist as inverse functions of integral expression containing Lambert $W$ functions [45].

Using Eqs. (A.2), closed formulae for the level set curves $g(x(t), y(t))=0$ can be obtained. Considering the equation

$$
\frac{d y}{d x}=\frac{y}{y+\frac{1}{3} \rho r_{\mathrm{t}}}\left(\rho r_{\mathrm{c}} x^{2}-d_{\mathrm{c}}\right),
$$


variables can be separated and both sides of the resulting expression can be integrated to obtain

$$
\left[\eta+\frac{1}{3} \rho r_{\mathrm{t}} \log (\eta)\right]_{\eta=y_{0}}^{\eta=y(t)}=\left[\frac{1}{3} \rho r_{\mathrm{c}} \xi^{3}-d_{\mathrm{c}} \xi\right]_{\xi=x_{0}}^{\xi=x(t)} .
$$

Recalling that the previous substitutions imply that $x(t)=V(t)^{1 / 3}$ and $y(t)=$ $-\frac{1}{3} \rho k C(t)$, the expression in Eq. (13) is found.

A similar result for the level sets in the simpler case $C(t) \geq 1$ can be achieved by integrating the analogous separable differential equation

$$
\frac{d C}{d x}=\frac{\left(\rho r_{\mathrm{c}} x^{2}-d_{\mathrm{c}}\right) C}{\frac{1}{3} \rho\left(r_{\mathrm{t}}-k\right)}
$$

obtained after performing the usual substitution $x(t)=V(t)^{1 / 3}$. No substitution for $C(t)$ is necessary in this case. The level set curves are given by

$$
\frac{1}{3} \rho\left(r_{\mathrm{t}}-k\right) \log (C(t))-\frac{1}{3} \rho r_{\mathrm{c}}\left(V(t)-V_{I}\right)+d_{\mathrm{c}}\left(V(t)^{1 / 3}-V_{I}^{1 / 3}\right)=0,
$$

and can be inverted to obtain

$$
C(t)=\exp \left(\frac{\frac{1}{3} \rho r_{\mathrm{c}}\left(V(t)-V_{I}\right)-d_{\mathrm{c}}\left(V(t)^{1 / 3}-V_{I}^{1 / 3}\right)}{\frac{1}{3} \rho\left(r_{\mathrm{t}}-k\right)}\right),
$$

where $V_{I}$ is the $V$-coordinate at the intersection of the general solution with the line $C=1$ on the right side of the centre, as illustrated in Fig. 10. Note that, for this reason, this expression does not depend on $C_{0}$ or $V_{0}$, as was the case in Eq. (12).

\section{Appendix B. Accounting for T-cell infiltration of the tumour}

Although the model presented in this work assumes that the size of tumours is small and cancers have no blood supply, T-cells may still be able to infiltrate in between tumour cells even in the absence of blood vessels. For a partial exploration of this issue we replace the factor $\rho k V(t)^{2 / 3}$ in Eq. 1, which asserts T-cell attack proportional to tumour surface area, by $k V(t)$, which prescribes attack proportional to tumour volume. This revised model represents a limiting case with the most effective T-cell attack. Note also that the chemical signals produced 
by the T-cells on the surface of or outside the tumour during the attack diffuse away more easily than any produced by a T-cell that has penetrated the tumour, so it is reasonable to retain area-dependence in the term that models the growth of the leukocyte population. These assumptions lead to the following modified equations:

$$
\begin{aligned}
& \frac{d V}{d t}=\rho r_{\mathrm{t}} V(t)^{2 / 3}-k V(t) \min \{1, C(t)\}, \\
& \frac{d C}{d t}=\rho r_{\mathrm{c}} V(t)^{2 / 3} C(t)-d_{\mathrm{c}} C(t) .
\end{aligned}
$$

Note that $k$ has now different units than those discussed in Table 1, but the other parameters are unchanged. For the case of high coverage, i.e. $C(t)>1$, solutions can be found explicitly, and the tumour volume turns out to be:

$$
V(t)=\frac{e^{-k t}\left(e^{\frac{C_{1} k}{3}}+\rho r_{\mathrm{t}} e^{\frac{k \rho t}{3}}\right)^{3}}{k^{3}},
$$

where $C_{1}$ is an integration constant that depends on the initial tumour volume at $t=0$. Note that this constant is chosen differently than the $V_{I}$ in Eq. (11). Eq. (B.3) shows an exponential functional dependence on $t$, presents an absolute, single minimum at finite times and is unbounded for $t \rightarrow \infty$.

The solution for $C(t)$ is more elaborate, and is given by

$$
C(t)=C_{2} \alpha(t) \exp \left[\left(\frac{\rho^{3} r_{\mathrm{c}}}{k^{2}} r_{\mathrm{t}}^{2}-d_{\mathrm{c}}\right) t\right],
$$

where $C_{2}>0$ is an integration constant, and

$$
\alpha(t)=\exp \left[-\frac{\rho r_{\mathrm{c}}}{2 k^{3}}\left(e^{\frac{2}{3} k\left(C_{1}-t\right)}+12 \rho r_{\mathrm{t}} e^{\frac{1}{3} k\left(C_{1}-t\right)}\right)\right] .
$$

Again, this is a different result to Eq. (12). At large times $\alpha(t) \rightarrow 1$, and the behavior of $C(t)$ depends solely on the sign of the exponential in Eq. (B.4). For values of the tumour growth parameter $r_{\mathrm{t}}$ such that

$$
r_{\mathrm{t}}<\frac{k}{\rho^{3 / 2}}\left(\frac{d_{\mathrm{c}}}{r_{\mathrm{c}}}\right)^{1 / 2}=r_{\mathrm{t}}^{*}
$$

$C(t)$ is bounded. This condition is analogous to the condition $r_{\mathrm{t}}<k$ for Eqs. (1)(2), providing an upper bound on the tumour growth speed for a finite solution. 
If the coverage is smaller than 1, Eqs. (B.1)-(B.2) are not explicitly solvable, and no strategy can be found to produce an analytic solution. Transformations similar to those described in Appendix A are ineffective and level set curves do not exist. In fact it turns out that, for the meaningful case $r_{\mathrm{t}}<r_{\mathrm{t}}^{*}$, a spiral is present, with coordinates given by

$$
\left(V_{\mathrm{sp}}, C_{\mathrm{sp}}\right)=\left(\left(\frac{d_{\mathrm{c}}}{\rho r_{\mathrm{c}}}\right)^{3 / 2}, \frac{r_{\mathrm{t}}}{k}\left(\frac{r_{\mathrm{c}}}{d_{\mathrm{c}}}\right)^{1 / 2} \rho^{3 / 2}\right) .
$$

If $r_{\mathrm{t}}>r_{\mathrm{t}}^{*}$ no spiral (or any stable equilibrium) exists, analogously to Fig 3. If we compare Eqs. (6) and (B.5) we see that in changing our model by replacing areadepedent attack to volume-dependent attack, the $V$-coordinate of the equilibrium point is unchanged, but the $C$-coordinate acquires a pre-factor $\rho^{3 / 2}\left(r_{\mathrm{c}} / d_{\mathrm{c}}\right)^{1 / 2}$. The eigenvalues associated with $\left(V_{\mathrm{sp}}, C_{\mathrm{sp}}\right)$ are given by $\lambda_{1-2}=\alpha \pm i \omega$, where $\alpha=\tau / 2$ and $\omega=\sqrt{\Delta-\tau^{2} / 4}$, with $\tau$ being the trace and $\Delta$ the determinant of the Jacobian for Eqs. (B.1)-(B.2) evaluated at $\left(V_{\mathrm{sp}}, C_{\mathrm{sp}}\right)$ [44] and for $C(t)<1$. These quantities are given by:

$$
\begin{aligned}
& \tau=-\frac{1}{3} \rho^{\frac{3}{2}}\left(\frac{r_{\mathrm{c}}}{d_{\mathrm{c}}}\right)^{\frac{1}{2}} r_{\mathrm{t}}, \\
& \Delta=\frac{2}{3} \rho^{\frac{3}{2}}\left(r_{\mathrm{c}} d_{\mathrm{c}}\right)^{\frac{1}{2}} r_{\mathrm{t}} .
\end{aligned}
$$

This equilibrium point is always a spiral, because $\tau<0$ due to all model parameters being positive. A depiction of typical orbits starting from different initial conditions is given in Fig. B.13. There are no other equilibria present except for the trivial, unstable, degenerate cases $(V, C)=(0,0)$ and $(V, C)=\left(0,\left(\rho r_{\mathrm{t}} / k\right)^{3}\right)$.

In conclusion, no eradication solution is present for $r_{\mathrm{t}}<r_{\mathrm{t}}^{*}$ in Eqs. (B.1)-(B.2). After a number of damped oscillations that depend on the initial conditions $V(0)$ and $C(0)$ and the chosen values of parameters, the tumour volume $V(t)$ and the coverage $C(t)$ reach an equilibrium and perpetually maintain the constant values $\left(V_{\mathrm{sp}}, C_{\mathrm{sp}}\right)$. This scenario represents an eternally static "dormant" tumour, which is incapable of growing or shrinking, and does not regrow [53]. Eqs. (B.1)-(B.2) show the importance of surface terms for the successful destruction of small, solid cancers, in the context of our model. According to this formulation, the limiting case of a volume-proportional term for the interaction between cancer cells and leukocytes does not produce eradication. 


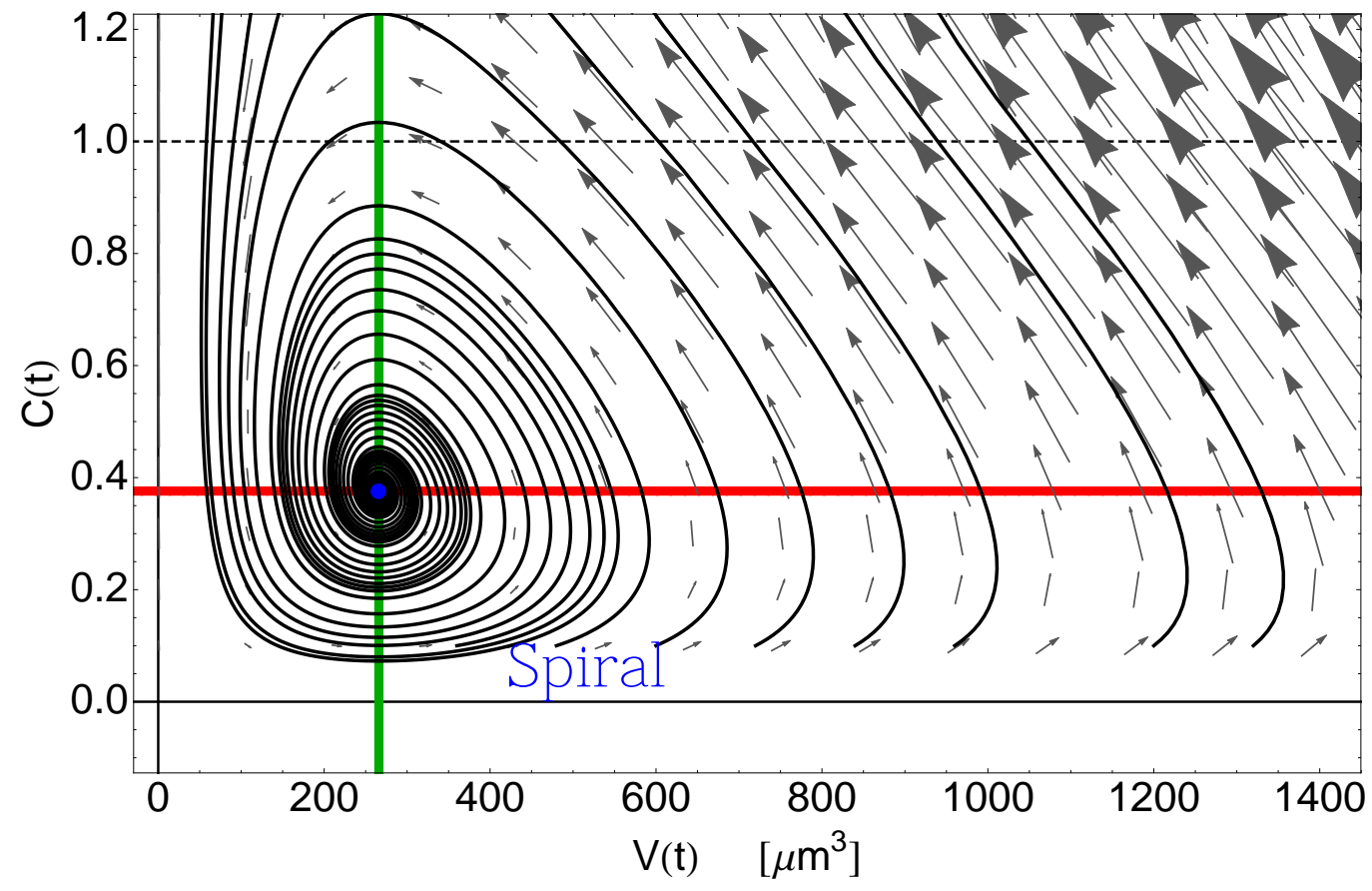

Figure B.13: Example of typical solutions spiralling to $\left(V_{\mathrm{sp}}, C_{\mathrm{sp}}\right)$ for $r_{\mathrm{t}}<r_{\mathrm{t}}^{*}$. No eradication solution is possible: tumours are perpetually dormant. Parameters are given by $r_{\mathrm{t}}=0.1 \mu \mathrm{m}$. day $^{-1}, k=0.2$ day $^{-1}, d_{\mathrm{c}}=0.2$ day $^{-1}, r_{\mathrm{c}}=0.001 \mu \mathrm{m}^{-2} \cdot$ day $^{-1}$. Note that $k$ has different units than those discussed in Table 1. 


\section{University Library}

\section{- M M I E E R VA A gateway to Melbourne's research publications}

Minerva Access is the Institutional Repository of The University of Melbourne

Author/s:

Frascoli, F;Kim, PS;Hughes, BD;Landman, KA

Title:

A dynamical model of tumour immunotherapy

Date:

2014-07-01

Citation:

Frascoli, F., Kim, P. S., Hughes, B. D. \& Landman, K. A. (2014). A dynamical model of tumour immunotherapy. MATHEMATICAL BIOSCIENCES, 253 (1), pp.50-62. https:// doi.org/10.1016/j.mbs.2014.04.003.

Persistent Link:

http://hdl.handle.net/11343/58830 